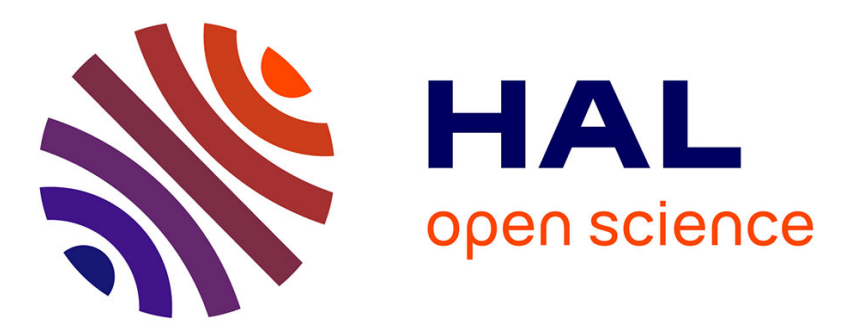

\title{
Influence of the components of propagule pressure, Allee effects, and stochasticity on the time to establish introduced populations
}

\author{
Nicolas Bajeux, Frédéric Grognard, Ludovic L. Mailleret
}

\section{- To cite this version:}

Nicolas Bajeux, Frédéric Grognard, Ludovic L. Mailleret. Influence of the components of propagule pressure, Allee effects, and stochasticity on the time to establish introduced populations. Journal of Theoretical Biology, 2019, 471, pp.91-107. 10.1016/j.jtbi.2019.03.012 . hal-02139554v2

\section{HAL Id: hal-02139554 \\ https://hal.inria.fr/hal-02139554v2}

Submitted on 14 Mar 2022

HAL is a multi-disciplinary open access archive for the deposit and dissemination of scientific research documents, whether they are published or not. The documents may come from teaching and research institutions in France or abroad, or from public or private research centers.
L'archive ouverte pluridisciplinaire HAL, est destinée au dépôt et à la diffusion de documents scientifiques de niveau recherche, publiés ou non, émanant des établissements d'enseignement et de recherche français ou étrangers, des laboratoires publics ou privés. 


\title{
Influence of the components of propagule pressure, Allee effects, and stochasticity on the time to establish introduced populations
}

\author{
Nicolas Bajeux $^{\mathrm{a}, \mathrm{b}}$, Frédéric Grognard ${ }^{\mathrm{a}}$, Ludovic Mailleret ${ }^{\mathrm{a}, \mathrm{c}}$ \\ ${ }^{a}$ Université Côte d'Azur, Inria, INRA, CNRS, Sorbonne Université, Biocore team, \\ Sophia Antipolis, France \\ ${ }^{b}$ University of Manitoba, Department of Mathematics, Winnipeg, Canada \\ ${ }^{c}$ Université Côte d'Azur, INRA, CNRS, ISA, France
}

\begin{abstract}
Forecasting whether individuals of an introduced population will succeed to establish is a challenge in invasion and conservation biology. The present work aims to decouple the impact of the components of propagule pressure on the time for population establishment in the presence of Allee effects and stochasticity in propagule sizes. The mean first passage time (MFPT) for a population to reach a viable size is used as a measure of the establishment success for the introduction processes involving periodic introductions. By fixing the introduction rate (mean number of introduced individuals per unit time) and varying the period of introduction from small ranges (small and frequent introductions) to large ones (infrequent and large releases), we study the influence of introduction distribution over time. These patterns of introduction are compared in a semi-stochastic model by observing which factors minimize the MFPT from an initially absent population, and hence, ensure the fastest population establishment. We investigate the influence on these minima of the introduction rate, variability in the introduction sizes, and occurrence of catastrophes that temporarily wipe out the population. Whereas most investigated cases show that infrequent and large introductions favor population establishment as expected, small and frequent introductions are preferred when the introduction rate is large and/or the variability in the introduction size is strong. Moreover, we observed counterintuitively that catastrophes strongly increase MFPT at small periods of introduction. In addition, we showed that stochasticity in introduction tends to increase the MFPT except when the introduction rate is small and introductions are
\end{abstract}


evenly spread out in time.

Keywords: Colonization, Biological Invasion, Impulsive Differential Equations, Mean First Passage Time

\section{Introduction}

Most studies on introduced populations aim to understand how and why propagules succeed or fail to establish in a new habitat. Many studies have identified major factors that greatly influence the probability that an introduced population is successfully established (Blackburn et al., 2011). Among those factors, the intensity and deployment of population introductions in space and time have been identified as key predictors of establishment success (Simberloff, 2009). In nature, population introductions do not usually consist of a single introduction event, but instead, of multiple releases of organisms. Multiple introductions are indeed prevalent in invasions by alien species (Dlugosch and Parker, 2008). Multiple introductions are also common in population management, for instance in augmentative biological control (van Lenteren, 2000) or in reintroduction biology (Armstrong and Seddon, 2008) programs. Propagule pressure is a composite measure that characterizes the different facets of the introduction effort (Blackburn and Duncan, $2001)$; it has been recognized as the best predictor of population establishment Lockwood et al. (2005). Two key components of propagule pressure have been identified Simberloff (2009): propagule size or introduction size, which corresponds to the number of individuals introduced per introduction events, and propagule frequency, which is simply the frequency of introduction events. An increase in either the propagule size or frequency is generally positively associated with establishment success because both increase the overall introduction effort.

The probability of establishment is also greatly impacted by intraspecific interactions occurring during the establishment of the incipient population. Allee effects are now considered as important, if not common mechanisms that influence the dynamics of ecological populations (Dennis et al., 2016). Since introduction schemes essentially involve small populations (Deredec and Courchamp, 2007; Drury et al., 2007), Allee effects may play a crucial role in the dynamics of introduced populations (Taylor and Hastings, 2005; Tobin et al., 2011). Strong demographic Allee effects refer to the dynamics of a population that becomes extinct below a certain critical size (Brassil, 
2001; Wang and Kot, 2001; Boukal and Berec, 2002). Building on this threshold behavior in an explicitly spatial context, theoretical investigations have explored how Allee effects alter biological invasions (Taylor and Hastings, 2005). By studying the persistence of metapopulations, viability thresholds were identified for populations inhabiting variable patchy environments Amarasekare, 1998; Bascompte, 2003). A lower speed of spread of invaders has also been reported in the presence of Allee effects in the invading species (Lewis and Kareiva, 1993), inducing time lags during which the population does not increase or spread (Taylor and Hastings, 2005; Drake and Lodge, 2006). Therefore, recent investigations stipulate that the management of invasive species needs to consider Allee effects (Taylor and Hastings, 2005; Tobin et al., 2011).

In addition to Allee effects, introduced populations may be subject to stochastic perturbations. Environmental and demographic stochasticity can affect population dynamics and may be the cause of population extinction (Dennis, 2002). For instance, catastrophic events, an extreme form of environmental stochasticity that drastically reduces a population (Shaffer, 1987; Lande, 1993), may play a crucial role in determining the persistence of populations (Lande et al., 2003). Moreover, stochasticity can also affect the introduction process itself, causing variations in the sizes or timing of introductions (Rajakaruna et al., 2013; Drake et al., 2015). Despite its probable pervasive influence, the effect of variability in propagule pressure components on population establishment has not been actively investigated to date, but see (Rajakaruna et al., 2013; Lewis et al., 2016). Exploring the consequences of different forms of stochasticity on population establishment is, therefore, relevant in an introduction biology context (Drake and Lodge, 2006; Drury et al., 2007; Rajakaruna et al., 2013; Potapov and Rajakaruna, 2013).

Few studies have investigated the influence of propagule size and frequency on establishment success in the presence of demographic Allee effects and stochastic perturbations during the introduction process. Whereas experimental studies in absence of Allee effects have shown that small and frequent introductions increase the probability of establishment (Drake et al., 2005; Hedge et al., 2012), large propagules appear to enhance establishment success in the presence of Allee effects in both empirical and theoretical studies. For instance, a recent field mesocosm experiment by Sinclair and Arnott (2016) demonstrated that for a given introduction rate, larger propagules lead to higher probabilities of establishment in a sexually reproducing mysid that is likely affected by Allee effects. Mathematical investigations also in- 
dicate that the probability of establishment increases with propagule size in populations that are influenced by Allee effects (Dennis, 2002; Drake and Lodge, 2006). Furthermore, numerical simulations have revealed that in the presence of strong demographic Allee effects, infrequent and large introductions lead to faster invasions (Wittmann et al., 2014; Mailleret and Lemesle, 2009) or to a larger probability of establishment (Drolet et al., 2016).

However, the presence of stochasticity complicates the matter. It is generally acknowledged that larger propagule sizes favor establishment in the presence of stochasticity and Allee effects (Liebhold and Bascompte, 2003, Simberloff, 2009; Fauvergue et al., 2012). However, some modeling studies did not observe a clear link between stochasticity and establishment probability and concluded that environmental stochasticity had little influence compared to Allee effects (Drolet et al., 2016). Actually, in the presence of environmental stochasticity alone, frequent introductions appear to perform better than rarer and larger ones (Simberloff, 2009; Fauvergue et al., 2012). Using branching process models Haccou and Iwasa (1996) showed that in the presence of environmental perturbations, multiple small size introductions increase introduction success compared to a single large one. In a comparable scenario, Drolet et al. (2016) also evidenced a positive link between propagule frequency and establishment probability, but it was only observed at small frequencies.

The impact of intrinsic population dynamics with Allee effects and perturbations induced by population introductions and environmental stochasticity on population establishment, therefore, merits further investigations. In this study, we propose a semi-stochastic model in which intrinsic population growth is deterministically described by an ordinary differential equation, while stochastic perturbations occur as discrete events. Perturbations correspond to introduction events, which induce population size increases, and to catastrophic events, which cause a temporary extinction of the population until the next introduction event. We assume that introductions occur periodically, with continuously distributed introduction sizes, and that catastrophic events occur randomly during the introduction process. Population establishment is considered successful once the population size exceeds a given target size, at which it is deemed safe from Allee effects and catastrophic events. We study the performance of an introduction program via the mean first passage time (MFPT) required for a population to exceed this target from a population of size zero (Drury, 2007; Wittmann et al., 2014). We explore the impact of the propagule pressure pattern (determined by the 
period between two introduction events) on the MFPT to identify whether frequent and small introductions or rare and large ones are preferable in terms of optimizing the introduction process (i.e. making the MFPT as low as possible) for a given global introduction effort. We study how the MFPT and the optimal introduction patterns are affected by the introduction rate (mean number of introduced individuals per time unit) and the target population size. Further, we investigate how the variance in introduction sizes and the rate at which catastrophic events occur influence these quantities. To perform these analyses, we first demonstrate that in the proposed setting, the MFPT required to reach the target population size from any non-negative initial population size can be computed as a function that is a solution of an integral equation. We then simulate this solution for the different parameter settings considered via a numerical approach based on a method of successive approximations.

\section{Modeling framework}

We assume that intrinsic population growth is continuous in time while events such as introductions or catastrophes occur as discrete events in time. Such features require a hybrid modeling framework. The different components of the resulting impulsive system are first detailed. Then, stochastic factors are described and finally, the overall introduction process is defined.

\subsection{Semi-discrete modeling}

The population growth is modelled using an ordinary differential equation. Let $y(t)$ be the population size at time $t$, we therefore have:

$$
\dot{y}(t)=f(y(t)) .
$$

We introduce strong demographic Allee effects into the intrinsic population growth function $f($.$) by assuming that there exists a so called Allee threshold$ $K_{a}>0$. The population goes extinct below this threshold (i.e. $f(y)<0$, for $y \in\left(0, K_{a}\right)$ and $\left.f(0)=0\right)$ while it grows when the population size is larger than this threshold, at least for $y$ close to $K_{a}$, (i.e. $f\left(K_{a}\right)=0$ and $\left.f^{\prime}\left(K_{a}\right)>0\right)$.

Such a general function $f($.$) provides some robustness for mathematical$ proofs and developments. For simulations, we will use the strong demo- 
graphic Allee effect model proposed by Gruntfest et al. (1997) and Courchamp et al. (1999), which can be expressed as:

$$
\dot{y}=r y\left(1-\frac{y}{K}\right)\left(\frac{y}{K_{a}}-1\right),
$$

with $r>0$ is the so-called intrinsic population growth rate, $K>0$ is the carrying capacity and $0<K_{a}<K$ is the Allee threshold, under which extinction of the population occurs when introductions do not take place. These parameters are summarized in Table 1.

Introduction events are successively made in time, and they instantaneously increase the population density to a higher level. A discrete-time equation represents such events and is generically formalized as follows:

$$
y\left(t_{i}^{+}\right)=y\left(t_{i}\right)+h_{i}, i \in \mathbb{N}^{*},
$$

where number $i$ represents the $i^{\text {th }}$ introduction event and time $t_{i}$ represents the instant at which this introduction event occurs. At this point, a quantity $h_{i}$ of individuals is introduced. The $t_{i}^{+}$notation depicts the time right after $t_{i}$, so that the population is instantaneously increased at the introduction event (Mailleret and Lemesle, 2009). Note that there is no introduction at time $t=0$.

Such impulsive introductions have been studied in a fully deterministic framework in Mailleret and Grognard (2006, 2009); Nundloll et al. (2010); Bajeux et al. (2017). They studied periodic introductions of individuals based on the following equation:

$$
y\left(i T^{+}\right)=y(i T)+\mu T, i \in \mathbb{N}^{*},
$$

with $\mu$ is the introduction rate, $T$ is the period at which individuals are introduced so that $t_{i}=i T$, and $\mu T$ is the number of introduced individuals at each introduction event. For a given introduction rate, varying the period $T$ allows us to investigate a full set of propagule pressure patterns, ranging from small and frequent introductions to large and rare ones.

In this work, we retain the periodic introduction pattern, but we consider that introductions are not precisely constant in size and are affected by random perturbations that lead to stochasticity in introduction size. We also consider that large stochastic changes in the environment may occur, 
that can randomly decimate the population in time. These catastrophes are modeled through the following discrete equation:

$$
y\left(t_{j}^{+}\right)=0, j \in \mathbb{N}^{*},
$$

in which the $t_{j}$ times are the instants at which the $j^{\text {th }}$ catastrophe event occurs.

\subsection{Stochastic factors}

Propagule pressure can be described as a Poisson process of intensity $\mu$ that captures the transport process from source to destination and the individual probability of survival (Jerde and Lewis, 2007). In this respect, Potapov and Rajakaruna (2013) proposed a continuous-time stochastic differential equation to describe introduction stochasticity in which the introduction rate has a variance equal to its mean. Merging a generalized form of this approach and the introduction rate format of Mailleret and Grognard (2006, 2009), we arrive at introduction sizes in a distribution with a mean $\mu T$ and a variance proportional to the mean: $v=c_{p} \mu T$, where $c_{p}$ is a coefficient of proportionality. We incorporated these features in a log-normal distribution with a mathematical form that is detailed in Appendix A.

We further assume that catastrophes occur following a Poisson process of intensity $\lambda_{c}$, so that the time between two consecutive catastrophes follows an exponential distribution of parameter $\lambda_{c}$. Therefore, the probability that at least one catastrophe occurs during a period of time $T$ is $p_{c}=1-e^{-\lambda_{c} T}$. Two or more catastrophes during one period do not impact the population further since it will be at size 0 until the next introduction event.

\subsection{Models}

Population dynamics throughout the introduction process are represented by intrinsic population dynamics that are disrupted by two kinds of perturbations: introduction events and catastrophes that obey discrete equations. The generic resulting impulsive model is as follows:

$$
\left\{\begin{array}{l}
\dot{y}(t)=f(y(t)), t \neq i T, t_{j} \\
y\left(i T^{+}\right)=y(i T)+h_{i}, \\
y\left(t_{j}^{+}\right)=0 .
\end{array}\right.
$$




\begin{tabular}{lll}
\hline Demographic & Definition & Values \\
\hline$r$ & Intrinsic growth rate & 1 \\
$K_{a}$ & Allee threshold & 1 \\
$K$ & Carrying capacity & 10 \\
$K_{T}$ & Target size & $1,1.1,2,5$ \\
\hline Introduction & \\
\hline$\mu$ & Mean introduction rate & $0.2,0.3,0.5,2$ \\
$T$ & Introduction period & {$[0.1,10]$} \\
$\mu T$ & Mean of introduction size & - \\
$c_{p}$ & Coeff. of proportionality for variance & {$[0.001,1]$} \\
$c_{p} \mu T$ & Variance of introduction size & - \\
\hline Catastrophe & & \\
\hline$\lambda_{c}$ & Poisson parameter & $0,0.1,0.2$ \\
$p_{c}$ & probability over one period & $p_{c}=1-e^{-\lambda_{c} T}$
\end{tabular}

Table 1: Parameters used for modeling the introduction process, their definition and values used in the simulations of the Results section.

The latter model is the aggregation of Equations (1), (3) and (5) and it has been used to perform our mathematical analysis in the most general case. A more specific impulsive model is used for simulations. It is given by:

$$
\left\{\begin{array}{l}
\dot{y}=r y\left(1-\frac{y}{K}\right)\left(\frac{y}{K_{a}}-1\right), t \neq i T, t_{j} \\
y\left(i T^{+}\right)=y(i T)+h_{i}, \text { with } h_{i} \sim \log \mathcal{N}(\alpha, \beta) \\
y\left(t_{j}^{+}\right)=0, \text { with }\left(t_{j+1}-t_{j}\right) \sim \operatorname{Exp}\left(\lambda_{c}\right) .
\end{array}\right.
$$

Figure 1 illustrates an introduction process using model (7). The process starts with an initial density of the population $y_{0}=0.2$. Periodic introductions are performed based on a period $T=0.5$ and an introduction rate $\mu=0.3$. The population density before the $i^{t h}$ release is given by $y(i T)$, $i>0$ with, for example, $y(T)$ and $y(2 T)$ on the figure. The post-release densities are $y\left(i T^{+}\right)=y(i T)+h_{i}$. Figure 1 illustrates the first passage time, which is the time at which the target size $K_{T}$ is crossed for the first time. A catastrophe occurs at the approximate time $t=2.2$. 


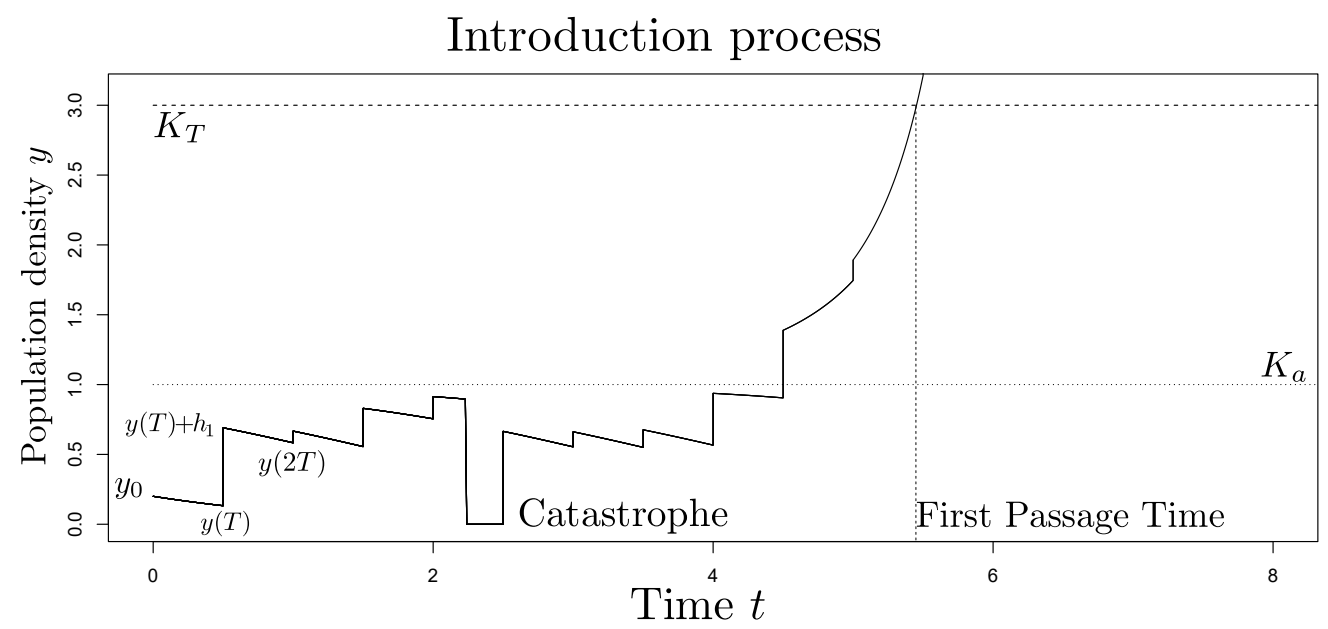

Figure 1: Population dynamics based on model (7). The dynamics used are given by (2), with $r=1, K_{a}=1$ and $K=100$. The mean introduction rate is $\mu=0.3$, the period of introduction is $T=0.5$ and the coefficient of proportionality between the mean and the variance is given by $c_{p}=0.1$. The target size is $K_{T}=3$.

\section{Measure to quantify success of establishment}

Since a stochastic representation for introduction sizes is used, the probability of a successful introduction is always 1 over a sufficiently long period of time. It is therefore not an appropriate measure to quantify introduction success. Thus, we consider the MFPT, which defines an average timescale for a stochastic event to first occur (Van Kampen, 1992; Polizzi et al., 2016), as a better measure to quantify the success of an introduction process (Drury et al., 2007).

\subsection{Integral equation of the MFPT without catastrophes}

MFPT for continuous processes that are disrupted by shot noises, i.e. random jumps that instantaneously change the level of the process, have been formulated in terms of integral equations by Masoliver (1987) without catastrophe consideration. Using a similar theoretical approach but keeping a general form concerning the distribution of introduction sizes, we obtain an integral equation in terms of the MFPT. We define $\Gamma\left(y_{0}\right)$ as the MFPT required for a population of initial size $0 \leqslant y_{0} \leqslant K_{T}$ to cross the target 
size $K_{T}$. Assuming that population dynamics follow Equations (6) without catastrophes, we determined by first step analysis (see Appendix B for its detailed derivation) that the MFPT is a solution of the implicit equation:

$$
\Gamma\left(y_{0}\right)=\theta\left(y_{0}\right)+\int_{0}^{K_{T}-y\left(\theta\left(y_{0}\right)\right)} \chi\left(h_{1}\right) \Gamma\left(y\left(\theta\left(y_{0}\right)\right)+h_{1}\right) d h_{1},
$$

with $\theta\left(y_{0}\right)=\min \left(T, \tau\left(y_{0}\right)\right)$, where $\tau\left(y_{0}\right)$ is the time it would take for the solution to reach the target size $K_{T}$ through population demography only. Therefore, $\tau\left(y_{0}\right)=+\infty$ when $y_{0}<K_{a} \leq K_{T}$ since population dynamics drive the population to extinction in that case. Function $\chi(h)$ is the probability density function that an introduction of size $h$ will occur.

There are two distinct terms on the right-hand size of (8). The first one is the timing of the next event. If $\tau\left(y_{0}\right) \leq T$, the target is reached through population dynamics before any introduction is performed. This occurs at time $\theta\left(y_{0}\right)=\tau\left(y_{0}\right)$ and the integral term is null since $K_{T}-y\left(\theta\left(y_{0}\right)\right)=0$, so that $\Gamma\left(y_{0}\right)=\tau\left(y_{0}\right)$. If $\tau\left(y_{0}\right)>T, \theta\left(y_{0}\right)=T$ and a discrete introduction event takes place at time $T$, so that the MFPT from $y_{0}$ is equal to the time $T$ plus the average time it would take to reach the target size from $y(T)$. This average lies in the integral term which sums the MFPT values $\Gamma\left(y(T)+h_{1}\right)=$ $\Gamma\left(y\left(T^{+}\right)\right)$multiplied by the probability density of an introduction of size $h_{1}$, $\chi\left(h_{1}\right)$; note that this integral is truncated at $h_{1}=K_{T}-y(T)$ since, for larger values of $h_{1}, y(T)+h_{1}>K_{T}$ and the target is exceeded at time $T^{+}$.

\subsection{Including catastrophes}

Catastrophic events are not considered in Equation (8). To include them, we propose that catastrophic events occur randomly during the process such that they wipe out the population present in the habitat of interest. The previously obtained MFPT changes since the population density randomly returns to zero when a catastrophe occurs before an introduction event. Hence, the integral equation of the MFPT becomes:

$$
\begin{aligned}
\Gamma\left(y_{0}\right) & =e^{-\lambda_{c} \theta\left(y_{0}\right)}\left(\theta\left(y_{0}\right)+\int_{0}^{K_{T}-y\left(\theta\left(y_{0}\right)\right)} \chi\left(h_{1}\right) \Gamma\left(y\left(\theta\left(y_{0}\right)\right)+h_{1}\right) d h_{1}\right) \\
& +\left(1-e^{-\lambda_{c} \theta\left(y_{0}\right)}\right)\left(T+\int_{0}^{K_{T}} \chi\left(h_{1}\right) \Gamma\left(h_{1}\right) d h_{1}\right) .
\end{aligned}
$$

The MFPT given in (9) is split into two terms. The first one represents the scenario where no catastrophe occurs before $\theta\left(y_{0}\right)$ and the second one 
a scenario where it does occur. The first term is the probability that no catastrophe occurs before $\theta\left(y_{0}\right), e^{-\lambda_{c} \theta\left(y_{0}\right)}$, which simply multiplies a MFPT factor built just as in Equation (8). The second term depicts the situation where a catastrophe happens before $\theta\left(y_{0}\right)$; the next event is therefore an introduction since the population is null after the catastrophe and $f(0)=$ 0 . Hence, the second term is the probability that at least one catastrophe occurs before $T, 1-e^{-\lambda_{c} \theta\left(y_{0}\right)}$, which simply multiplies a MFPT factor built with $\theta\left(y_{0}\right)=T$ and $y(T)=0$; in the end, this second term is equal to $\Gamma(0)$ multiplied by the probability that a catastrophe occurs before $\theta\left(y_{0}\right)$ : $\left(1-e^{-\lambda_{c} \theta\left(y_{0}\right)}\right) \Gamma(0)$.

\subsection{Successive approximations}

Equations (8) and (9) are similar to the Volterra integral equations, so that we use similar methods to compute solutions of these equations. The method of successive approximations (Polyanin and Manzhirov, 2008, Shestopalov and Smirnov, 2002) is a recursive method for solving integral equations using (8) or (9) as recurrence equations to obtain the next iteration. We simply have to fix a first approximative function denoted $\Gamma_{0}$ and compute the next ones until they converge to a fixed point. Basically, using Equation (8), the initial approximation $\Gamma_{0}\left(y_{0}\right)$ and the following approximations are given by:

$$
\begin{aligned}
& \Gamma_{0}\left(y_{0}\right)=\theta\left(y_{0}\right) \\
& \Gamma_{i+1}\left(y_{0}\right)=\theta\left(y_{0}\right)+\int_{0}^{K_{T}-y\left(\theta\left(y_{0}\right)\right)} \chi\left(h_{1}\right) \Gamma_{i}\left(y\left(\theta\left(y_{0}\right)\right)+h_{1}\right) d h_{1},
\end{aligned}
$$

for all $y_{0} \in\left[0, K_{T}\right]$. We show convergence of these successive approximations to the MFPT $\Gamma\left(y_{0}\right)$ in Appendix $\mathrm{C}$.

For a given introduction period $T$, the MFPT values for all initial densities of the population from $y_{0}=0$ up to $y_{0}=K_{T}$ with a step of 0.02 are computed. Indeed, since the $y\left(\theta\left(y_{0}\right)\right)+h_{1}$ term in the integral of the MFPT can assume a value from 0 to $K_{T}$, it is mandatory to have access to such values at each step of the recursive approximation. A trapezoidal rule is used to compute the numerical integration and a linear interpolation is performed to obtain the term $\Gamma_{i+1}\left(y\left(\theta\left(y_{0}\right)\right)+h_{1}\right)$ from the previous approximation $\Gamma_{i}($.$) .$ 


\section{Results}

The method of successive approximations given by equation (10) allows for the numerical computation of the MFPT given by Equations (8) and (9). Introduction rates, target levels, and stochastic parameter values have been explored to elucidate how they impact the MFPT as a function of the introduction period.

\subsection{Outputs}

By numerically computing successive approximations of the MFPT for a range of values of $T$, we compute contour plots as shown in Figure 2, This figure gives the MFPT on a 2-dimensional space in which the horizontal axis is the initial population size $y_{0}$ and the vertical axis is the period of each introduction. Figure 2 is obtained line by line: for each value of the period $T$ from 0.1 to 3.5 , we compute the algorithm as described by equation (10) until convergence occurs. The bottom lines of both sub-figures are precisely computed. Indeed, as $T \rightarrow 0$, System (7) without catastrophic event has the same dynamical behavior as the following equation:

$$
\dot{y}=r y\left(1-\frac{y}{K}\right)\left(\frac{y}{K_{a}}-1\right)+\mu,
$$

which represents deterministic population growth with a continuous introduction process at a rate of $\mu$. Using Equation (11), the First Passage Time for the population to cross $K_{T}$ for all the range of initial density $y_{0}$ is then computed and included in the contour plot as the MFPT to crosses $K_{T}$ for $T=0$.

One way to read and extract information from Figure 2 is to fix a period of introduction (i.e. consider the horizontal lines). This observation of the MFPT is intuitive and straightforward since the MFPT is decreasing with the initial density, for all parameter sets, as expected.

The plots can also be read by considering a vertical line (i.e. for a fixed initial density $y_{0}$ ), which shows how the MFPT changes with respect to the period of introduction $T$. This is directly related to the pattern of introductions. Since the introduction rate is fixed, a small $T$ represents a high frequency of small size introductions, while large $T$ describe low-frequency introductions of large sizes.

In Figure 2a, the consideration of the vertical line for $y_{0}=0$, shows that the population is likely to establish fastest if the period of introduction is 

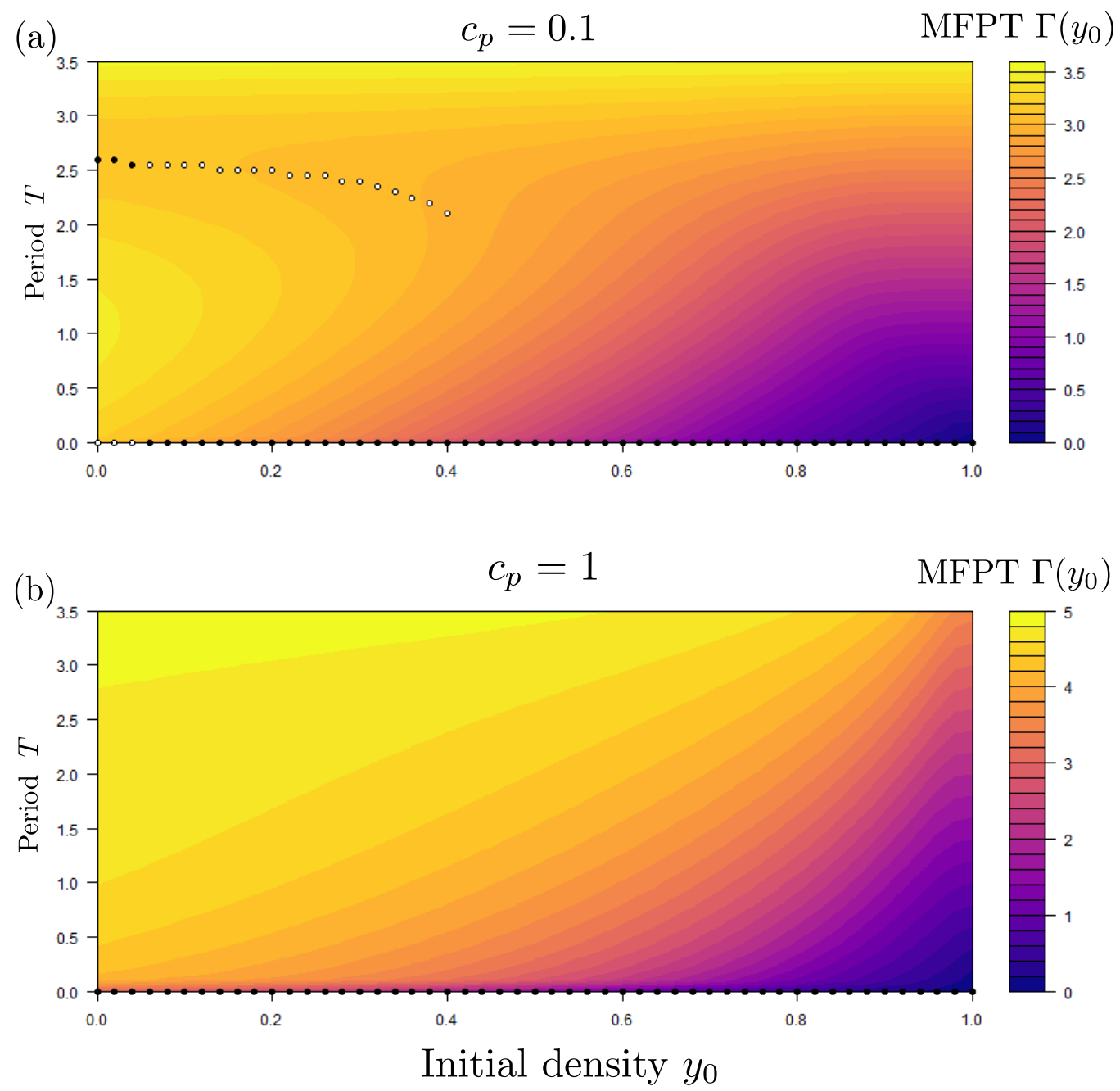

Figure 2: Contour plots representing the mean first passage time (MFPT) for the population to cross $K_{T}=K_{a}=1$ for different values of $y_{0}$ and $T$. The initial density $y_{0}$ varies from 0 to $K_{T}$ while period $T$ varies from 0 to 3.5. The introduction rate is fixed at $\mu=0.5$ in both sub-figures, the coefficient of proportionality between $\mu T$ and the variance is $c_{p}=0.1$ for (a) and $c_{p}=1$ for (b). No catastrophe occurs during the introduction process. The global minimum of the MFPT as a function of $T$, for a given $y_{0}$, is described by the black circles, while the white circles represent a local minimum.

approximately 2.7 units of time. However, Figure $2 \mathrm{~b}$, which is computed for a larger coefficient $c_{p}$, shows that small and frequent introductions lead to 
the fastest establishment of the population. Such differences in results are discussed later by comparing and analyzing different sets of parameters.

By fixing the initial population $y_{0}$, it is thus possible to obtain the minima of the MFPT as a function of the period of introduction $T$, i.e. identify the optimal introduction pattern. The circle curves in Figure 2 describe how these minima evolve, with the black circles referring to the global minimum while the white circles represent a local minimum. In Figure $2 a$, the global minimum is obtained for large periods, i.e., infrequent and large introductions, when the initial density is low $\left(y_{0} \leq 0.06\right)$. In the same range of initial densities, a local minimum is also present at $T=0$. The global minimum becomes a local one for intermediate initial densities $\left(0.06<y_{0} \leq 0.4\right)$ and the global minimum lies at $T=0$. This induces a change of the optimal pattern from large periods, i.e. infrequent and large introductions, to very small periods, i.e. small and frequent introductions. Once the initial density becomes large $\left(y_{0}>0.4\right)$, the local minimum disappears. In Figure $2 \mathrm{~b}$, for any initial population, the optimal strategy is to frequently introduce individuals, since the circle line is at $T=0$.

A common feature of all the contour plots we obtained is that there always exists a critical initial density above which the positive global or local minima disappears, as in Figure $2 a$, and the only global minimum is then for $T=0$. Whereas such a feature is interesting for the introduction schemes starting at different initial densities, the present study focuses on the MFPT for $y_{0}=0$, i.e. the worst initial density for the population.

\subsection{Influence of target size and introduction rate}

In Figure 3, we explore the response of the MFPT from $y_{0}=0$ as a function of the introduction period $T$ for different values of the target size $K_{T}$ from $K_{T}=K_{a}=1$ to $K_{T}=5$, three values for the introduction rate $\mu=0.3$, $\mu=0.5$ and $\mu=2$, and little variability in the introduction size, i.e. for

$c_{p}=0.01$. Each curve is obtained by taking the values along the vertical axis of a corresponding contour plot defined as in Figure 2. The MFPT cannot be smaller than the period $T$ since the target cannot be reached before one introduction happens. This defines an inaccessibility zone in Figure 3, which is illustrated by the gray area; when the MFPT is at the upper boundary of this zone, establishment succeeds through a single introduction. For $T \rightarrow 0$, the value of the MFPT is computed via the continuous dynamics given by equation (11) and plotted with dots near the vertical axis. To assess the performance of the successive approximation algorithm, we also computed 
the curves obtained in Figure 4 using the Monte method Carlo (see Appendix E). These simulations confirm the validity of the successive approximation method, based on a much heavier computational load.

We observe that the MFPT is increasing with the target size $K_{T}$ for all $T$, which is natural since the population size has to reach a higher level to be successful. The same is true for the main impact of the introduction rate $\mu$, which decreases the MFPT when it increases. Less trivial is the impact of $\mu$ on the optimal introduction pattern. When a small introduction rate is considered ( $\mu=0.3$ in Figure $3 \mathrm{a}$ ), the population establishes faster when the period of introduction is at a relatively large value, corresponding to a crossing of $K_{T}$ based on precisely one introduction. For small values of $T$, a local minimum is observed at $T=0$, which is identical to the situation that is shown on the vertical axis of Figure 2a. This pattern becomes more apparent when the introduction rate is larger, (Figure $3 \mathrm{~b}$ ) and finally, the MFPT may increase globally for large introduction rates (Figure 3k). Thus, the global minima observed for large periods of introduction disappear as the introduction rate becomes larger, giving rise to the situation illustrated along the vertical axis of Figure $2 \mathrm{~b}$.

MFPT curves as a function of $T$ oscillate as the introduction rate is increased. This results from the whole number of introductions needed to cross $K_{T}$. Indeed, when there is no variability in introduction sizes, we observe a sawtooth-shape of the MFPT curves: a sharp decrease in the MFPT appears when a whole number of introductions is required to exactly reach $K_{T}$. This phenomenon is illustrated in Figure D.7 in Appendix D, which represents the same MFPT curves as in Figure 3 without stochasticity $\left(c_{p}=0\right)$. In Figure 3, a relatively small variance in the introduction size has been used for simulations, but the pattern is only present when the introduction rate is large enough (Figures 3b and 3c). In Figure 3a, the target size is crossed after many introductions, which leads to a smoothing of the MFPT curves. Since $\mu$ is larger in figures $3 \mathrm{~b}$ and $3 \mathrm{k}$, less introduction events are needed to cross $K_{T}$ and the pattern observed when no variability occurs becomes more apparent.

Since the population is initially extinct, it is not possible for the population to reach the target size before the first introduction event. This time range is described by the shaded areas in Figure 3. It is the region under the curve $\Gamma(0)=T$ that represents the mean time for the population to cross the target size at the first introduction event. 

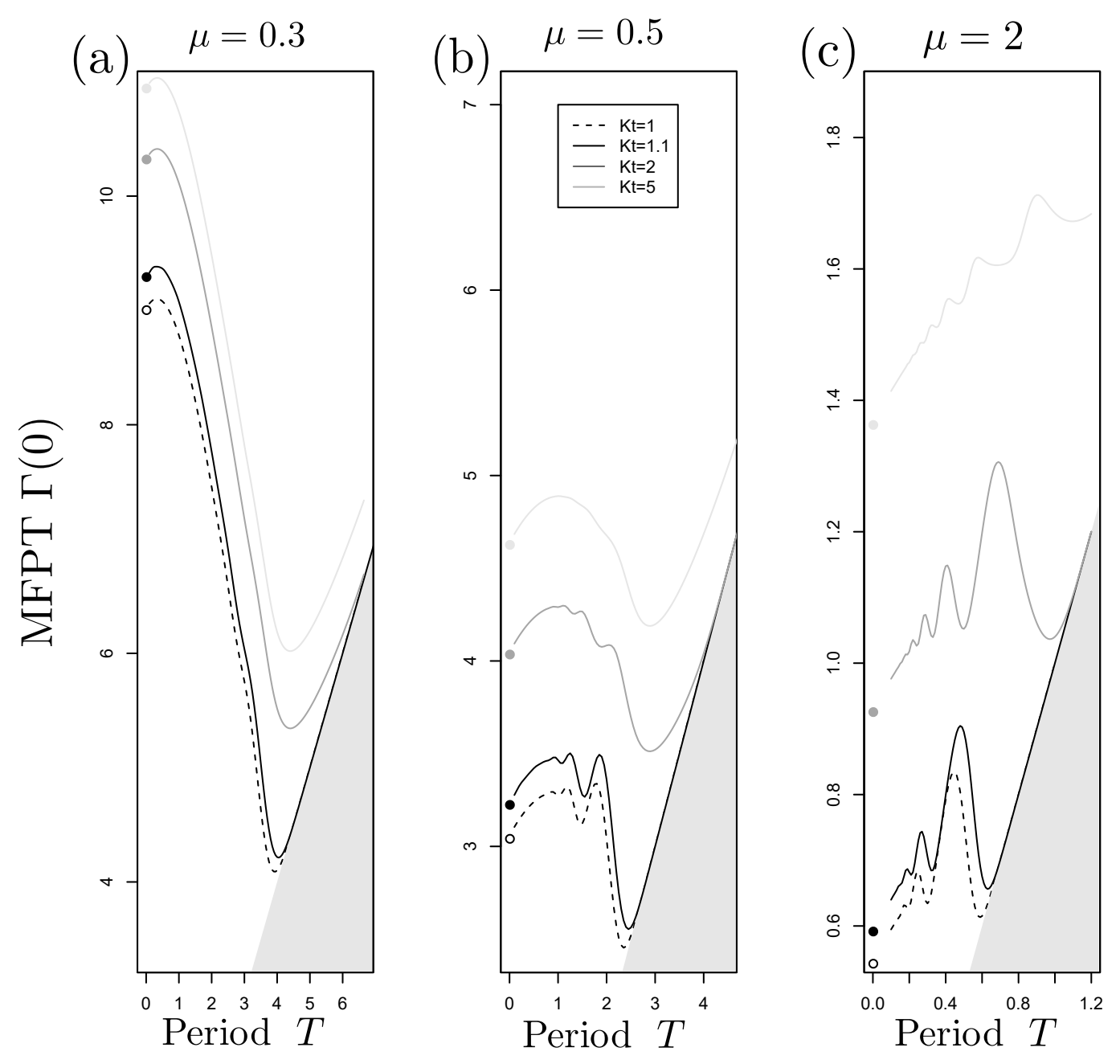

Figure 3: Influence of $\mu$ and $K_{T}$ : Mean first passage time (MFPT) for population size to cross $K_{T}$ from $y_{0}=0$ as a function of the introduction period $T$. (a) Describes the MFPT for $\mu=0.3$, (b) is obtained using $\mu=0.5$ and (c) for $\mu=2$. Results for four values of $K_{T}$ are shown: $K_{T}=1$ (dashed), $K_{T}=1.1$ (solid black), $K_{T}=2$ (dark gray) and $K_{T}=5$ (light gray). Dots denote the continuous introduction $T \rightarrow 0$ obtained from Model (11). The variability in the introduction size is $c_{p}=0.01$. The period $T$ varies from 0 to 7 in (a), to 5 in (b), and to 1.2 in (c). MFPT is unachievable in the shaded areas. 


\subsection{Variability in introduction sizes}
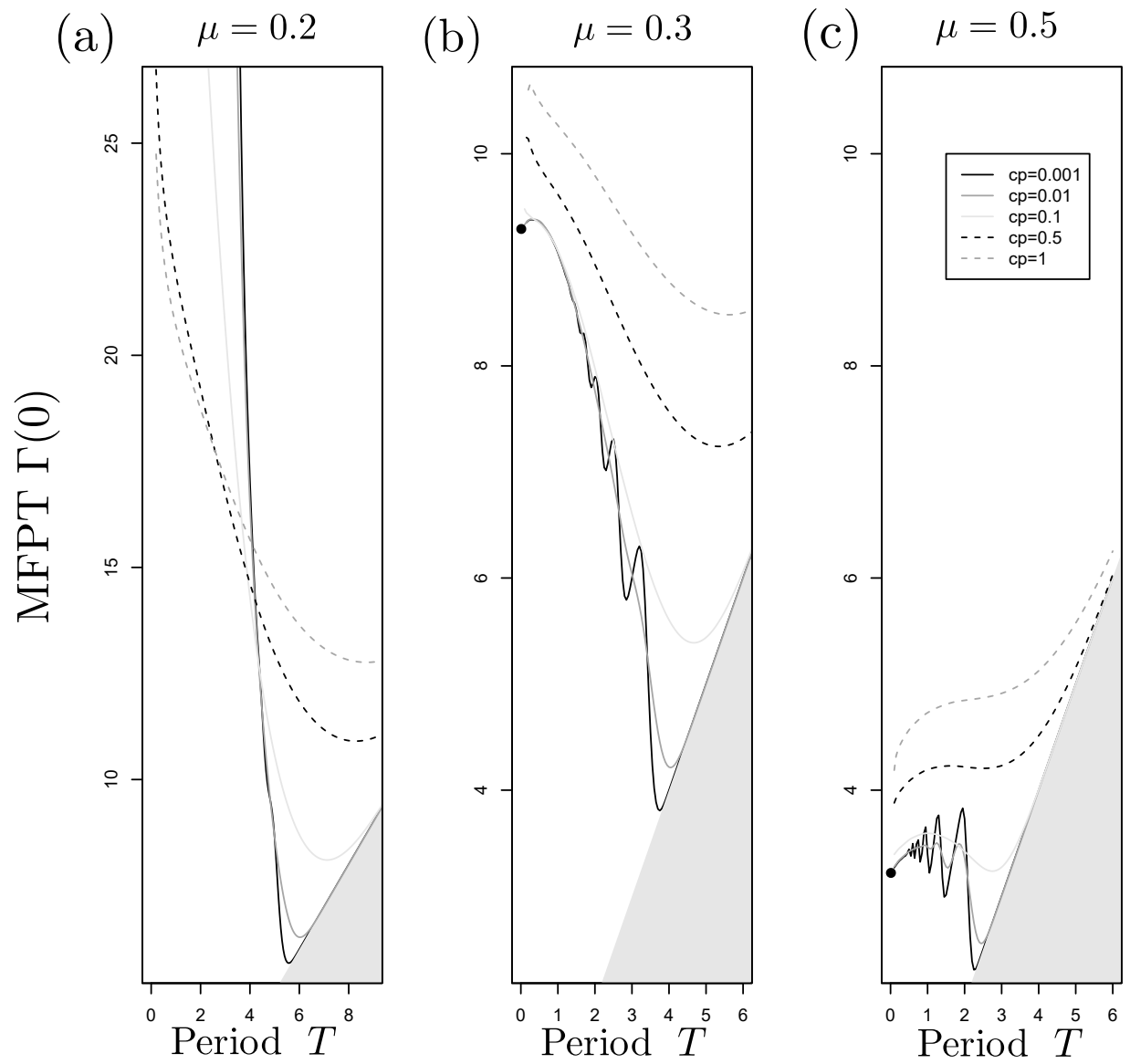

Figure 4: Influence of $\mu$ and $c_{p}$ : Mean first passage time (MFPT) using the method of successive approximation for different values of the coefficient of proportionality between the variance and the mean introduction size. The MFPT is given with respect to the period of the introduction for a fixed value of $y_{0}=0$. Period $T$ varies from 0 to 10 in sub-figure (a) and from 0 to 6 in both sub-figures $\mathrm{b}$ and $\mathrm{c}$. The introduction rate $\mu$ is 0.2 in (a), 0.3 in (b) and 0.5 in (c). The target size $K_{T}$ is equal to 1.1 in all sub-figures. Solid and dashed curves describe the MFPT for different variability in the introduction sizes for all sub-figures. Solid black, dark and light gray give the MFPT for small variances with $c_{p}=0.001, c_{p}=0.01$ and $c_{p}=0.1$, respectively, while the dashed black and dark gray give the MFPT for larger variances using $c_{p}=0.5, c_{p}=1$. The point described the MFPT computed with Model (11). MFPT is not reachable in shaded areas (mean time faster than MFPT in one introduction).

As shown in Figure 4 we investigate the response of the MFPT as a 
function of the introduction period $T$ to different amounts of variability in introduction sizes $\left(c_{p}=0.001\right.$ up to $\left.c_{p}=1\right)$ for three different introduction rates $\mu=0.2, \mu=0.3$ and $\mu=0.5$.

The main result is that, for sufficiently large introduction rates (Figure $4 \mathrm{~b}$ and $\mathrm{c}$ ), the MFPT mostly increases with $c_{p}$. At very small ranges of the introduction rate (Figure 4 a), stochasticity in introduction size favors small and frequent introductions while, for larger values of $\mu$ (Figure $4 \mathrm{~b}$ and 4c), stochasticity promotes large and infrequent introductions. When the variance is very small (i.e. $c_{p}=0.001$ and $c_{p}=0.01$ ), the oscillating pattern observed in Figure 3 is apparent but disappears as $c_{p}$ increases.

When $\mu$ is small (Figure 4 and b), a global minimum is observed for large periods of introduction. This global minimum is such that the population size crosses $K_{T}$ with approximately one introduction event on average. The optimal period increases with variability, which is quite natural since small introduction sizes induced by the stochasticity have to be overcome. However, when $\mu$ is large (Figure 4c), the global minima occur for large periods only when there is little variability in the introduction size (black and dark gray solid curves). When $c_{p}$ increases (dashed curves), the global minima are shifted towards $T=0$ and the MFPT increases in $T$. This means that when introduction size is random, introducing individuals more frequently promotes population establishment. This pattern is attributable to overdispersion and the positive skewness of the distribution of introduction sizes when $c_{p}$ is large and the expected introduction size is small Appendix A. Indeed, positive skewness implies that the determined introduction sizes are often very small but occasionally much larger. Such large introductions allow then to overshoot the Allee threshold in one step and subsequently, the target population size.

The presence of stochasticity affects the MFPT differently when $\mu$ is very small (Figure 4a). When introductions are rare and large, high levels of stochasticity in introduction sizes are detrimental to population establishment, as previously indicated. However, when periods of introduction are small, higher stochasticity in the introduction sizes reduces the time for establishment. Bajeux et al. (2014) pointed out that there exists a critical value of $\mu$ depending on $T$ below which population establishment is not possible in an entirely deterministic introduction process, and $\mu=0.2$ is below that level since it cannot compensate for the fastest population decay rate. For $c_{p} \leq 0.01$, the introduction process is very similar to such a deterministic introduction process, leading the MFPT to become very large. For larger $c_{p}$ 
values, there is a more frequent occurrence of larger than expected introductions that can compensate for the fastest decay rate, which leads to a faster population establishment.

Though it is not clear in Figure 4 , because of the heavy numerical burden associated with computing the MFPT for small values of $T$ using our algorithm, the MFPT converges to a single value when $T \rightarrow 0$ for any $c_{p}$ (black dot on the left of Figures $4 \mathrm{~b}$ and $4 \mathrm{c}$ ). The gap between the $\operatorname{dot}($ i.e. $T \rightarrow 0)$ and the first MFPT computed using the algorithm (i.e. $T=0.1$ ) becomes larger as variance in the introduction sizes increases. There is no such dot in Figure 4 a since, in a deterministic framework (equation (11)), population cannot be established, so that all curves go to $+\infty$ as $T \rightarrow 0$.

\subsection{Catastrophes}

Finally, based on Figure 5, we investigate the influence of catastrophe occurrences on the relation between the MFPT and the introduction period $T$, by considering three values of $\lambda_{c}\left(\lambda_{c}=0, \lambda_{c}=0.1\right.$ and $\left.\lambda_{c}=0.2\right)$ for two different variabilities in introduction sizes $\left(c_{p}=0.001\right.$ and $\left.c_{p}=1\right)$.

As expected, increasing the rate at which catastrophes occur increases the MFPT (Figures $5 \mathrm{a}$ and $5 \mathrm{~b}$ ). When the variance of the introduction size is very small (Figure 5a), optimal introduction patterns correspond to a large period (approximately $T=3$ ). This optimum value is reinforced as the environmental stochasticity increases.

For a large variance of introduction size $\left(c_{p}=1\right.$, Figure 5b) and without catastrophes, small and frequent introductions are optimal (dashed curve). However, the occurrence of catastrophes increases the MFPT, especially for small introduction periods. Consequently, the optimal introduction pattern switches back to large $T$ values as $\lambda_{c}$ increases. This result stems from the fact that when the introduction size is sufficiently large, catastrophes do not influence the MFPT, since a single introduction will often achieve population establishment. Further, because the MFPT is computed from an initial condition $y_{0}=0$, the occurrence of a catastrophe before the first introduction event has no impact because the population size is already null.

\section{Discussion}

The present work aims to decouple the impact of the components of propagule pressure on the time for population establishment in the presence of Allee effects and stochasticity. As suggested in empirical Sinclair 

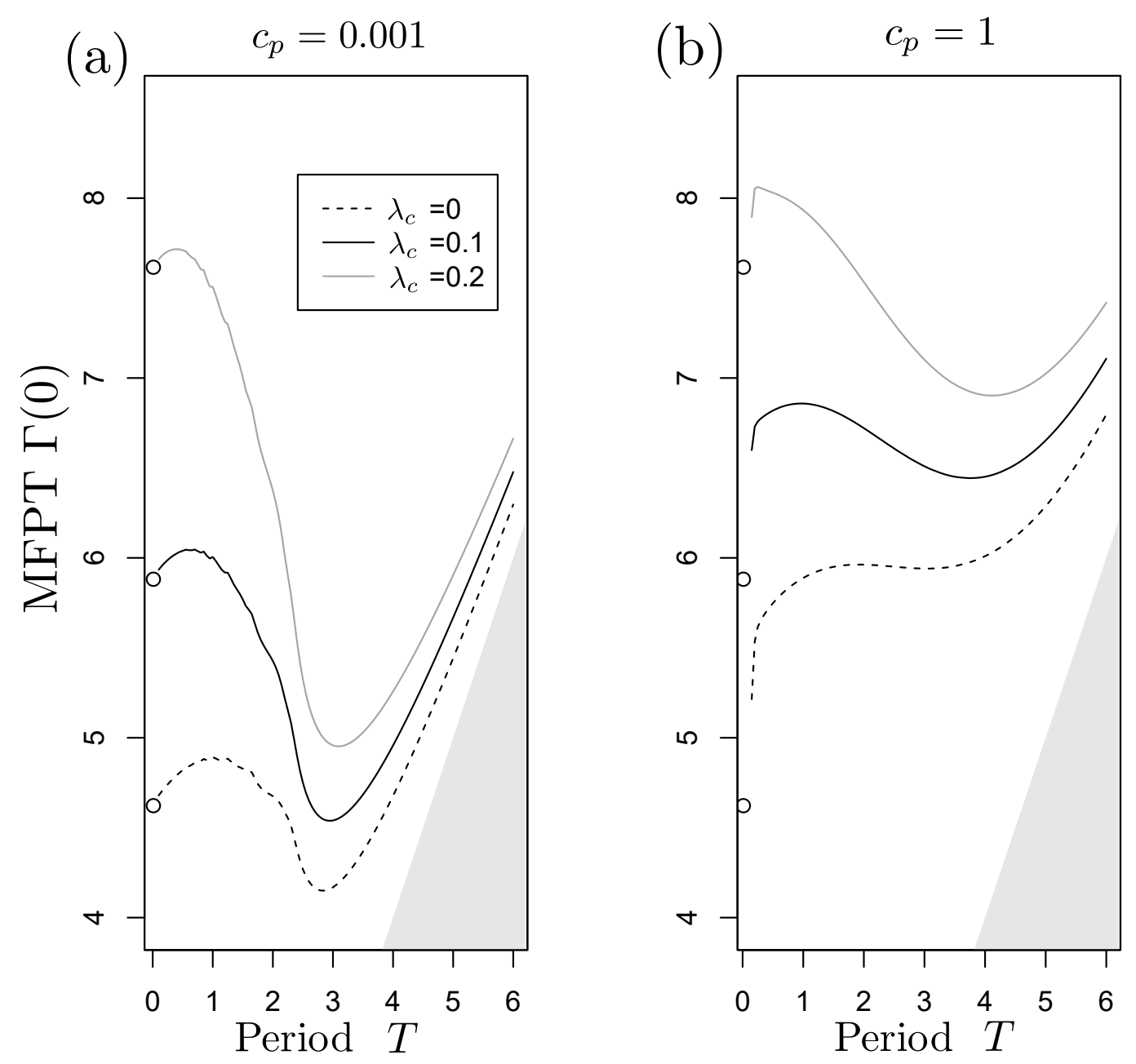

Figure 5: Influence of $c_{p}$ and $\lambda_{c}$ : Mean first passage time (MFPT) for the population to cross $K_{T}$ starting from an initial density $y_{0}=0$. The introduction rate $\mu$ is 0.5 , the period varies from 0 to 6 and the target size $K_{T}=5$ is in both sub-figures. In (a), a small variance is taken $c_{p}=0.001$ while a large one is represented in (b), $c_{p}=1$. Three values for the Poisson parameter $\lambda_{c}$ are chosen: $\lambda_{c}=0$ in dashed black, $\lambda_{c}=0.1$ in black and $\lambda_{c}=0.2$ in dark gray. Points represent the MFPT for $T \rightarrow 0$. MFPT is not reachable in shaded areas (mean time faster than MFPT in one introduction).

and Arnott, 2016), mathematical (Dennis, 2002, Drake and Lodge, 2006; Mailleret and Lemesle, 2009) and computational studies (Wittmann et al., 2014; Drolet et al., 2016), it has generally been determined that infrequent 


\begin{tabular}{cccc}
\hline \multicolumn{2}{c}{ Parameter values } & Best & Local best \\
\hline \multicolumn{1}{c}{$\mu$} & & \\
\hline Small & & Size & Frequency \\
Medium & & Size & $\times$ Frequency \\
Large & & Frequency & $\times$ Size \\
\hline$c_{p}$ & $\mu$ & & \\
\hline Small & Any & Size & $\times$ Frequency \\
Large & Small & Size & Frequency \\
& Large & Frequency & None \\
\hline$\lambda_{c}$ & $c_{p}$ & & \\
\hline Small & Small & Size & Frequency \\
& Large & Frequency & Size \\
Large & Any & Size & Frequency \\
\hline
\end{tabular}

Table 2: Main results obtained from previous figures. Best and local best strategies as a function of the parameter values are given for all studied cases. "Frequency" indicates that the best (resp. local best) strategy is to introduce individuals frequently using small introduction sizes. "Size" indicates that the best (resp. local best) strategy is to introduce individuals rarely with large introduction sizes. " $\times$ Frequency" indicates that there are multiple local minima with period of introduction that are smaller than the optimal value. " $\times$ Size" indicates that there are multiple local minima with a period of introduction that is greater than the optimal value.

and large introductions decrease the mean time for establishment in the presence of the Allee effects. However, we interestingly discovered some situations for which the optimal strategy, on the contrary, is based on small and frequent introductions (summary Table 2). This situation arises mainly when the introduction rate is large and/or when introduction sizes are very random. Furthermore, we counterintuitively observed that when environmental catastrophes resulting in extinction occurs too often, small and frequent introductions are particularly negatively impacted. These main findings are summarized in Table 2 .

\subsection{Introduction rate}

In our simulations, the introduction rate $\mu$ describes the effort during the overall process. Since it is the mean number of introduced individuals per 
time unit, an increase of the introduction rate leads to an increase of the introduction size, for the same period. It is therefore very natural that larger introduction rates lead to smaller values of the MFPT. Furthermore, when $\mu$ is large, even frequent introductions are sufficiently large to overcome the negative impact of the Allee effects and/or variability in the introduction sizes. These frequent introductions are therefore more efficient in terms of the time to establishment compared to rarer patterns of introduction (Figure $3 \mathrm{c}$ ). When $\mu$ is more limiting (Figure $3 a, b$ ), only infrequent, and thus large introductions can swiftly outweigh the effects of Allee and stochasticity, which leads to the classical result concerning the superiority of such introduction patterns in presence of Allee effects. These results highlight and confirm the primary importance of the introduction rate in driving populations to establishment (Blackburn and Duncan, 2001; Lockwood et al., 2005; Simberloff, 2009).

\subsection{Variability in introduction sizes}

The introduction rate also plays an important role when introduction sizes are variable. Indeed, when the introduction rate is large enough, small and frequent introductions outperform other patterns of introduction when strong stochasticity arises (Figure 4c). Hence, large fluctuations in introduction sizes tend to disadvantage intermediate and large periods of introductions more than smaller ones. This result holds because if a given, supposedly large introduction fails and is in fact small because of the stochasticity, there is a long time delay before the next large introduction. When introductions are small and frequent, failing one of them has little impact because the next release arises soon, and the missed quantity of the introduced individuals is small. Furthermore, the distribution of introduction sizes is increasingly positively skewed since the expected introduction size is small A.6 in Appendix A). Therefore, when small and frequent introductions are performed, many very small introductions are realized, but rarely, a large one occurs and drives the population over the Allee threshold. The population can then cross the target population size through intrinsic population growth. This advantage of the small and frequent introduction pattern becomes more sensible as the variability in introduction sizes increases since the skewness of the distribution of introduction sizes increases as well.

Two recent studies investigated the effects of stochasticity in immigration based on stochastic differential equation models (Rajakaruna et al., 2013. 
Potapov and Rajakaruna, 2013). Rajakaruna et al. (2013) considered favorable environments (positive intrinsic growth of the population) or situations in which Allee effects occur but the introduction rate was larger than the maximum population decay due to the Allee effects. In both cases, the authors showed that stochasticity in the introduction process tends to decrease the probability of establishment of the population. Potapov and Rajakaruna (2013) explored the influence of stochasticity in unfavorable environments when the introduction rate was smaller than the maximum population decay due to Allee effects on average. In such situations, they evidenced a positive stochasticity effect on the probability of population establishment. In the present study, we mainly considered the first situation, when $\mu$ is larger than the maximum of the population decay. Similar to Rajakaruna et al. (2013), we determine that stochasticity in the introduction sizes tends to hamper population establishment, for all types of introduction patterns (Figure 4). Simulation studies were performed to investigate the case when the introduction rate is smaller than the maximum population decay (Figure 4a). Just as Potapov and Rajakaruna (2013), we determine that when introductions are, on average, small and frequent, stochasticity in introduction sizes tends to favor population establishment. However, when the introduction pattern moves towards larger and infrequent ones, the association is reversed and higher stochasticity in the introduction size becomes detrimental to population establishment, as is the case for larger introduction rates. This is because since we have determined that stochasticity is not required for large introductions to transition across the region of fastest decay. Therefore, there appears to be a general negative association between population establishment and immigration stochasticity, except in the special cases where the immigration rate is rather small, and the introductions are evenly spread out over time.

\subsection{Environmental catastrophes}

The occurrence of environmental catastrophes that wipe out populations during the introduction process appear to slow down population establishment for all types of introduction patterns but has more impact on small and frequent ones (Figure 5). This is quite an unexpected result since it is often asserted that in the presence of environmental stochasticity, populations may face difficulties in recovering from negative conditions when individuals are introduced too rarely (Simberloff, 2009). However, it seems

to be in line with the results of recent modeling studies by Cassey et al. 
(2014) and Drolet et al. (2016), which show that performing many introductions in the presence of high environmental stochasticity actually hinders population establishment. Here, catastrophes do not affect population establishment before the first introduction event since the population is empty at that moment. Thus, the strategy which entails waiting before introducing large numbers of individuals is not hindered by catastrophes during this time period. On the contrary, strategies based on the gradual build-up of the population through more frequent and smaller introductions may rapidly face catastrophes, which completely resets the overall introduction process. Therefore, the occurrence of catastrophes is more influential over smaller and more frequent introduction patterns.

In this paper, we approach environmental stochasticity based on the rather extreme case of catastrophes that destroy the entire population at random moments in time. Yet, environmental stochasticity can also assume softer forms as temporal perturbations that affect the probability of mortality and natality in a population (Lande et al., 2003). Figure 2 (iii) from Cassey et al. (2014) shows that extreme forms of environmental perturbations impact the probability of establishment more than moderated perturbations. Also, they emphasize that, in the absence of spatial structure, the effect of this type of perturbations cannot be alleviated by spreading many small introductions over space, as in (Grevstad, 1999). Hence, in Cassey et al. (2014) and the present work that only considers localized populations, large perturbations directly impact the entire population which cannot escape, so that accounting for such perturbations is crucial in the evaluation of the success or failure of the introduction process.

Nevertheless, a deeper investigation of how temporal fluctuations in growth rate induced by milder environmental stochasticity affects the MFPT could be explored. At this point, our mathematical tools are not sufficiently developed to understand how such environmental perturbations could affect the MFPT. For instance, the consideration of minor catastrophes that do not wipe out the entire population but only a portion of it renders the problem of deriving the implicit integral equation defining the MFPT (Section 3.2 and Appendix B difficult and it remains an important challenge for future work in this regard.

\subsection{Comparison with the study by Wittmann et al. (2014)}

Wittmann et al. (2014) proposed a fully stochastic approach to compare MFPT to reach a target size under different temporal patterns of introduc- 
tion. Their study is very akin to the present one, with the major exception that the modeling frameworks are completely different. It is therefore relevant to point out the similarities and differences of the outputs between the two studies. Wittmann et al. (2014) considered different ecological scenarios in the function of the sign of the per capita growth rate of the population during the overall introduction process: Easy, Mixed, and Difficult ecological scenarios describe the per capita growth rates that on average are positive, negative, then positive, or entirely negative, as the population size grows. For easy scenarios, they determined that frequent introductions are likely to establish the population faster. We ran additional simulations using a logistic growth, i.e. with a positive growth rate during the overall introduction process, that confirm this point (not shown here). Mixed and difficult scenarios correspond in our study to target population sizes larger or equal to the Allee threshold, respectively. Wittmann et al. (2014) found that, in such scenarios, it is always better to rarely introduce individuals in large quantities. They argued that the variance in the introduction process is the key factor, in their model, that affects and tends to favor large introduction sizes. This is mostly in line with our findings, but not when the introduction rate and/or the variability in introduction sizes are large, for which small and frequent introductions are the most efficient (Figure $3 \mathrm{c}$ and $4 \mathrm{~b}$ ). Demographic stochasticity is not accounted for in our study and it may or may not be responsible for this apparent discrepancy between the two approaches. Therefore, we have determined that further research should be performed to investigate the performance of small and frequent introduction patterns in the presence of demographic stochasticity when the introduction rate and/or variability in introduction sizes are large.

\subsection{A note on the modeling framework}

Different choices were made to conceptualize the introduction process and to numerically simulate it. Measuring the success of population introduction poses the question of what is the best metric to quantify the success or failure of an introduced population. Four metrics have been identified: in empirical studies, both the abundance, i.e., the population size at the end of the experiment, and the probability of extinction are frequently used (Drake et al., 2005; Hedge et al., 2012; Sinclair and Arnott, 2016). In theoretical studies, both the first passage probability for the population to reach a target size before going extinct (Dennis, 2002; Drake and Lodge, 2006) and the MFPT for the population to reach a target size (Drury et al., 2007; Wittmann et al., 
2014) are regularly used. Here, abundance has been discarded since it can be strongly impacted by the intrinsic dynamics once the population is established. The second and third ones were not considered because we had the a priori that a population could become temporarily extinct, notably through environmental perturbations, without stopping the entire introduction process. This is because introductions occur repeatedly. Hence, we concentrated on the MFPT which provides quantitative information on the time to achieve success for an introduction process.

The choice of a log-normal distribution for model variability in introduction sizes was influenced by its positive support characteristic and versatility. The bell shape of the distribution was also considered to be representative of real-life variability in introduction size. An example of this is the fluctuations in propagule sizes or individual survival that can occur during actual introductions (see e.g. distributions of propagule sizes in Drake et al. (2015)). We also tested another form with a gamma distribution which essentially yielded similar qualitative results (not shown here). Nevertheless, it would be interesting to examine the influence of a distribution with a compact positive support, i.e. a distribution that would render introduction sizes between 0 and an absolute maximum size that could not be exceeded. Although such distributions would be substantially less flexible than the log-normal or gamma distribution, they may mitigate the effects of large variabilities in the introduction sizes on optimal introduction patterns. This is left as future works.

Finally, it could be possible to explore the influence of fluctuations in the timing of introductions, which were here considered to be periodic, since our theoretical framework includes stochasticity in the time interval between introductions (see Appendix B and Appendix C). Stochasticity in introduction timing, even without variability in the introduction size, may have different repercussions on the MFPT for population establishment. In particular, when catastrophes occur, the impact could become important if an introduction event becomes very rare because it allows the population to be sensitive to an environmental fluctuation for a longer period. These features combined with variation in the introduction sizes would certainly deserve further study to observe their relative influence on different introduction strategies. 


\section{Acknowledgements}

The authors acknowledge the support of the French Agence Nationale de la Recherche 664 (ANR) under grant ANR-13-BSV7-0011 (project FunFit) and of the SPE department of INRA (project ABCD).

\section{Appendix A. Probability Density Functions}

As indicated in Section 2, the variance of the introduction sizes is linear with the mean introduction size. We choose location and scale parameters $\alpha$ and $\beta$ of the log-normal distribution such that the variance of the introduction sizes is proportional to the mean introduction size :

$$
\begin{aligned}
& \alpha=\log \left(\frac{\mu T}{\sqrt{1+c_{p} /(\mu T)}}\right), \\
& \beta=\sqrt{\log \left(1+c_{p} /(\mu T)\right)} .
\end{aligned}
$$

Figure A.6 represents several probability density functions for different coefficients of proportionality, using a log-normal distribution. This is the distribution that has been selected for simulations in this investigation. The choice of the log-normal distribution was driven by its positive support and its similarity to the Gaussian distribution, at least for large means of introduction sizes (light gray in the figure). However, the log-normal distribution is skewed for small means and large variances. This difference appears in Figure A.6 in dark gray, black and dotted black, with an increasing magnitude. For the same mean of introduction size but for a larger variance, the mode of the density probability function is significantly different from the mean compared to the case with a small variance $c_{p}=0.001$ in light gray.

This skew changes the way introduction processes succeed. Indeed, a large skew implies that most of the introductions are small. However, a very large introduction sometimes has a big impact.

\section{Appendix B. Integral equation of the MFPT}

This section describes how to obtain the integral equation of the MFPT given by Equations (8) and (9). This is accomplished through the proof of the following theorem in a more general manner than that presented in 


\section{Probability Density Functions}

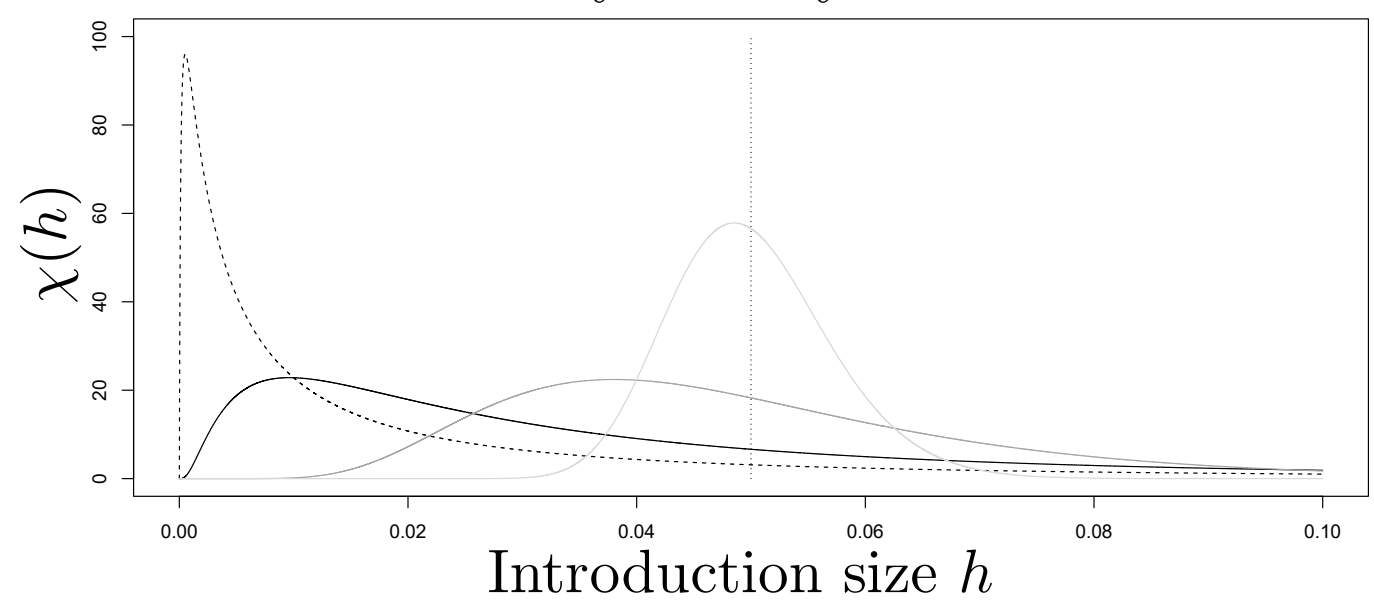

Figure A.6: Probability density functions for different values of the variance. The period of introduction is $T=0.1$, the introduction rate is $\mu=0.5$ and the coefficient $c_{p}$ varies between four values: $c_{p}=0.001$ (light gray), $c_{p}=0.01$ (dark gray), $c_{p}=0.1$ (black) and $c_{p}=1$ (dotted black).

the article, since introductions are made randomly in time and not just periodically. Thus, considering that the probability density function of time intervals is given by the function $\psi(t)$ and that time $\bar{\tau}_{i}$ denotes the time to cross $K_{T}$ using the intrinsic population growth, we established the following theorem, which is the main mathematical result of this work.

Theorem 1. Suppose that function $f$ satisfies the assumptions given in Section 2.1, the MFPT for the population to cross $K_{T} \geqslant K_{a}$ starting from an initial density $y_{0} \leqslant K_{T}$ is given as:

$$
\Gamma\left(y_{0}\right)=\theta\left(y_{0}\right)+\int_{0}^{\bar{\tau}_{1}} \psi\left(\tau_{1}\right) \int_{0}^{K_{T}-y\left(\theta\left(y_{0}\right)\right)} \chi\left(h_{1}\right) \Gamma\left(y\left(\theta\left(y_{0}\right)+h_{1}\right) d h_{1} d \tau_{1}\right.
$$

where

$$
\theta\left(y_{0}\right)=\min \left(\bar{\tau}_{1} \int_{\bar{\tau}_{1}}^{\infty} \psi\left(\tau_{1}\right) d \tau_{1}, \int_{0}^{\bar{\tau}_{1}} \tau_{1} \psi\left(\tau_{1}\right) d \tau_{1}\right) .
$$

Proof 1. To obtain (B.1), we first need to compute the probability to cross the target size $K_{T}$. To do so, we first obtain the probability $\bar{P}(i)$ to not 
cross $K_{T}$ during the $i^{\text {th }}$ interval, based on the knowledge that no crossing has occurred before:

$$
\bar{P}(i)=P\left(y_{i}+h_{i}<K_{T} \text { and } \tau_{i}<\bar{\tau}_{i} \mid\left(y_{l}+h_{l}<K_{T} \text { and } \tau_{l}<\bar{\tau}_{l}\right), \forall l<i\right) .
$$

Value of $\bar{P}(i)$ is

$$
\bar{P}(i)=\int_{0}^{\bar{\tau}_{i}} \psi\left(\tau_{i}\right)\left(\int_{0}^{K_{T}-y_{i}\left(\tau_{i}\right)} \chi\left(h_{i}\right) d h_{i}\right) d \tau_{i} .
$$

The probability that no crossing occurs with the growth of the population is $\int_{0}^{\overline{\tau_{i}}} \psi\left(\tau_{i}\right) d \tau_{i}$ and the probability to not cross $K_{T}$ from $y_{i}$ with a jump is $\int_{0}^{K_{T}-y_{i}} \chi\left(h_{i}\right) d h_{i}$. Since the population density $y_{i}=y\left(t_{i}\right)$ depends on the time at which the $i^{\text {th }}$ introduction occurs, the latter probability is inside the first integral representing the timing of the jump.

Then, the probability to cross $K_{T}$ during the $n^{\text {th }}$ interval by the growth of the population or by an introduction event is

$$
P(n)=P\left(y_{n}+h_{n}>K_{T} \text { or } t_{n}>t_{n-1}+\bar{\tau}_{n}\right)
$$

whose value is

$$
P(n)=\int_{\bar{\tau}_{n}}^{\infty} \psi\left(\tau_{n}\right) d \tau_{n}+\int_{0}^{\bar{\tau}_{n}} \psi\left(\tau_{n}\right) \int_{K_{T}-y_{n}}^{\infty} \chi\left(h_{n}\right) d h_{n} d \tau_{n} .
$$

The first integral is the probability that the $n^{\text {th }}$ introduction occurs after the crossing of $K_{T}$ because of the continuous growth of the population, while the second is the probability to cross the target size with an introduction event, which is only possible if no continuous crossing previously occurred. This is why there are two integrals to represent this probability. Additionally, note that when $y_{n-1}+h_{n-1} \leqslant K_{a}, \bar{\tau}_{n}=+\infty$, so that the first term disappears and the second simplifies to $\int_{K_{T}-y_{n}}^{\infty} \chi\left(h_{n}\right) d h_{n}$.

The probability density of the first passage time at time $t$ starting from an initial population density $y_{0}$ is defined as $p\left(t, y_{0}\right)$ such that

$$
p\left(t, y_{0}\right)=\sum_{n=1}^{\infty} p_{n}\left(t, y_{0}\right)
$$


where $p_{n}\left(t, y_{0}\right) d t$ is the probability that the population crosses the target size during the time interval $(t, t+d t)$, after $n-1$ introductions, and before or at the $n^{\text {th }}$ introduction. Thus, the MFPT is given by the integral notation

$$
\Gamma\left(y_{0}\right)=\int_{0}^{\infty} t p\left(t, y_{0}\right) d t
$$

Considering that the crossing of the population threshold occurs through population growth, thus, at time $t=\bar{\tau}_{n}+\sum_{i=1}^{n-1} \tau_{i}$, the probability density of an such event is given as:

$$
\bar{T}_{n}(t)=\delta\left(t-\left(\bar{\tau}_{n}+\sum_{i=1}^{n-1} \tau_{i}\right)\right)
$$

while the probability density of this event is 0 when $\bar{\tau}_{n}=+\infty$. So that, we define

$$
\bar{T}_{n}(t)=\left\{\begin{array}{l}
=\delta\left(t-\left(\bar{\tau}_{n}+\sum_{i=1}^{n-1} \tau_{i}\right)\right), \text { if } \overline{\tau_{n}} \text { is finite, } \\
=0, \text { otherwise. }
\end{array}\right.
$$

If the crossing of the population threshold occurs because of an introduction at time $t=\sum_{i=1}^{n} \tau_{i}$, the probability density of such an event is given as:

$$
T_{n}(t)=\delta\left(t-\sum_{i=1}^{n} \tau_{i}\right) .
$$

Then, using Equations (B.2), (B.3), (B.6) and (B.8), we obtain the probability density as:

$$
\begin{aligned}
& p_{n}\left(t, y_{0}\right)=\int_{0}^{\bar{\tau}_{1}} \psi\left(t_{1}\right) \int_{0}^{K_{T}-y_{1}} \chi\left(h_{1}\right)\left(\ldots \left(\int_{0}^{\bar{\tau}_{n-1}} \psi\left(\tau_{n-1}\right) \int_{0}^{\bar{K}_{T}-y_{n-1}} \chi\left(h_{n-1}\right)\right.\right. \\
& \left.\left.\left.\int_{\bar{\tau}_{n}}^{\infty} \psi\left(\tau_{n}\right) \bar{T}_{n}(t) d \tau_{n}+\int_{0}^{\bar{\tau}_{n}} \psi\left(\tau_{n}\right) \int_{K_{T}-y_{n}}^{\infty} \chi\left(h_{n}\right) T_{n}(t) d h_{n} d \tau_{n}\right) d h_{n-1} d \tau_{n-1}\right) \ldots\right) d h_{1} d \tau_{1}
\end{aligned}
$$

In the previous equation, the probability to not cross $K_{T}$ during the $n-1$ first introduction events is given by the $n-1$ first double integral of type 
$\int_{0}^{\bar{\tau}_{k}} \psi\left(\tau_{k}\right) \int_{0}^{K_{T}-y_{k}} \chi\left(h_{k}\right)$ as defined in $\mathrm{B} .2$. Then, the crossing of $K_{T}$ occurs at the $n^{\text {th }}$ introduction event at time $t_{n}=\sum_{i=1}^{n} \tau_{i}$ or during the $n^{\text {th }}$ period with the growth of the population at time $\bar{\tau}_{n}+\sum_{i=1}^{\infty} \tau_{i}$. Note that if $\bar{\tau}_{n}$ is infinite, a cross never occurs via population growth and $\bar{T}_{n}=0, \forall t$, and the integral $\int_{\bar{\tau}}^{\infty} \psi\left(\tau_{n}\right) \bar{T}_{n}(t) d \tau_{n}$ is equal to zero.

As in Masoliver (1987), we define the Laplace transform of $p_{n}\left(t, y_{0}\right)$ as:

$$
\hat{p}_{n}\left(s, y_{0}\right)=\int_{0}^{\infty} e^{-s t} p_{n}\left(t, y_{0}\right) d t
$$

and

$$
\hat{p}\left(s, y_{0}\right)=\sum_{n=1}^{\infty} \hat{p}_{n}\left(s, y_{0}\right) .
$$

Thus, using the last two equations and (B.5), we can show that:

$$
\Gamma\left(y_{0}\right)=-\left.\frac{\partial \hat{p}\left(s, y_{0}\right)}{\partial s}\right|_{s=0}
$$

and we also need to compute $\hat{p}\left(s, y_{0}\right)$ to obtain an expression for the MFPT.

From (B.9), it is possible to write $p_{n+1}\left(t, y_{0}\right)$ as a function of $p_{n}\left(t, y_{0}\right)$. Indeed,

$$
\begin{gathered}
p_{n+1}\left(t, y_{0}\right)=\int_{0}^{\bar{\tau}_{1}} \psi\left(t_{1}\right) \int_{0}^{K_{T}-y_{1}} \chi\left(h_{1}\right)\left(\ldots \left(\int _ { 0 } ^ { \overline { \tau } _ { n } } \psi ( \tau _ { n } ) \int _ { 0 } ^ { K _ { T } - y _ { n } } \chi ( h _ { n } ) \left(\int_{\bar{\tau}_{n+1}}^{\infty} \psi\left(\tau_{n+1}\right) \bar{T}_{n+1}(t) d \tau_{n+1}\right.\right.\right. \\
\left.\left.\left.+\int_{0}^{\bar{\tau}_{n+1}} \psi\left(\tau_{n+1}\right) \int_{K_{T}-y_{n+1}}^{\infty} \chi\left(h_{n+1}\right) T_{n+1}(t) d h_{n+1} d \tau_{n+1}\right) d h_{n} d \tau_{n}\right) \ldots\right) d h_{1} d \tau_{1}
\end{gathered}
$$

and we note in the latter equation that the terms from the first large parenthesis until the last one actually represent $p_{n}\left(y_{1}+h_{1}\right)$, we then obtain the following recurrence equation,

$$
p_{n+1}\left(t, y_{0}\right)=\int_{0}^{\bar{\tau}_{1}} \psi\left(\tau_{1}\right) \int_{0}^{K_{T}-y_{1}} \chi\left(h_{1}\right) p_{n}\left(t, y_{1}+h_{1}\right) d h_{1} d \tau_{1} .
$$

Thus, it is possible to obtain the probability density to cross $K_{T}$ at time $t$, starting from $y_{0}$, at the $(n+1)^{\text {th }}$ introduction or during the $(n+1)^{\text {th }}$ period based on the growth of the population as a function of the probability density 
to cross $K_{T}$ at time $t$, starting from $y_{1}+h_{1}$, at the $n^{\text {th }}$ introduction or during the $n^{\text {th }}$ period by the growth of the population. Then, we use this recurrence relation for the Laplace transform,

$$
\hat{p}_{n+1}\left(s, y_{0}\right)=\int_{0}^{\bar{\tau}_{1}} e^{-s \tau_{1}} \psi\left(\tau_{1}\right) \int_{0}^{K_{T}-y_{1}} \chi\left(h_{1}\right) \hat{p}_{n}\left(s, y_{1}+h_{1}\right) d h_{1} d \tau_{1},
$$

and apply it to obtain $\hat{p}\left(s, y_{0}\right)$ :

$$
\hat{p}\left(s, y_{0}\right)=\hat{p}_{1}\left(s, y_{0}\right)+\int_{0}^{\bar{\tau}_{1}} e^{-s \tau_{1}} \psi\left(\tau_{1}\right) \int_{0}^{K_{T}-y_{1}} \chi\left(h_{1}\right) \hat{p}\left(s, y_{1}+h_{1}\right) d h_{1} d \tau_{1},
$$

We obtain $\hat{p}_{1}\left(s, y_{0}\right)$ from the integration of $\mathrm{B} .9$ in $\mathrm{B} .10$ for $n=1$. We now have,

$$
p_{1}\left(t, y_{0}\right)=\int_{\bar{\tau}_{1}}^{\infty} \bar{T}_{1}(t) d \tau_{1}+\int_{0}^{\tau_{1}} \psi\left(\tau_{1}\right) \int_{K_{T}-y_{1}}^{\infty} \chi\left(h_{1}\right) T_{1}(t) d h_{1} d \tau_{1},
$$

so that,

$\hat{p}_{1}\left(s, y_{0}\right)=\int_{0}^{\infty} e^{-s t}\left(\int_{\bar{\tau}_{1}}^{\infty} \bar{T}_{1}(t) d \tau_{1}+\int_{0}^{\tau_{1}} \psi\left(\tau_{1}\right) \int_{K_{T}-y_{1}}^{\infty} \chi\left(h_{1}\right) T_{1}(t) d h_{1} d \tau_{1}\right) d t$.

Rearranging the terms and inverting the integrals, we obtain:

$$
\begin{aligned}
\hat{p}_{1}\left(s, y_{0}\right)= & \int_{\tau_{1}}^{\infty} \psi\left(\tau_{1}\right) \int_{0}^{\infty} e^{-s t} \bar{T}_{1}(t) d t d \tau_{1}+ \\
& \int_{0}^{\bar{\tau}_{1}} \psi\left(\tau_{1}\right) \int_{K_{T}-y_{1}}^{\infty} \chi\left(h_{1}\right) \int_{0}^{\infty} e^{-s t} T_{1}(t) d t d h_{1} d \tau_{1} .
\end{aligned}
$$

We now have two cases, either $\bar{\tau}_{1}$ is finite or infinite. When $\bar{\tau}_{1}$ is finite, that is when $y_{0}>K_{a}$, Equation (B.16) gives,

$$
\hat{p}_{1}\left(s, y_{0}\right)=\int_{\bar{\tau}_{1}}^{\infty} \psi\left(\tau_{1}\right) e^{-s \bar{\tau}_{1}} d \tau+\int_{0}^{\bar{\tau}_{1}} \psi\left(\tau_{1}\right) \int_{K_{T}-y_{1}}^{\infty} \chi\left(h_{1}\right) e^{-s \tau_{1}} d h_{1} d \tau_{1},
$$

because the integrals that depend on the Delta Dirac functions disappear.

$$
\frac{\partial \hat{p}_{1}\left(s, y_{0}\right)}{\partial s}=-\bar{\tau}_{1} \int_{\bar{\tau}_{1}}^{\infty} \psi\left(\tau_{1}\right) d \tau_{1}-\int_{0}^{\bar{\tau}_{1}} \tau_{1} \psi\left(\tau_{1}\right) e^{-s \tau_{1}} \int_{K_{T}-y_{1}}^{\infty} \chi\left(h_{1}\right) d h_{1} d \tau_{1}
$$


and the partial derivative of the function with respect to s of the second term of (B.15) is:

$$
\begin{aligned}
& -\int_{0}^{\bar{\tau}_{1}} \tau_{1} e^{-s \tau_{1}} \psi\left(\tau_{1}\right) \int_{0}^{K_{T}-y_{1}} \chi\left(h_{1}\right) \hat{p}\left(s, y_{1}+h_{1}\right) d h_{1} d \tau_{1} \\
& +\int_{0}^{\bar{\tau}_{1}} e^{-s \tau_{1}} \psi\left(\tau_{1}\right) \int_{0}^{K_{T}-y_{1}} \chi\left(h_{1}\right) \frac{\partial \hat{p}}{\partial s}\left(s, y_{1}+h_{1}\right) d h_{1} d \tau_{1} .
\end{aligned}
$$

Thus, by using $\hat{p}\left(0, y_{1}+h_{1}\right)=1$ and $(\mathrm{B} .12)$, we obtain

$$
\begin{aligned}
\Gamma\left(y_{0}\right) & =\bar{\tau}_{1} \int_{\bar{\tau}_{1}}^{\infty} \psi\left(\tau_{1}\right) d \tau_{1}+\int_{0}^{\bar{\tau}_{1}} \tau_{1} \psi\left(\tau_{1}\right) d \tau_{1} \\
& +\int_{0}^{\bar{\tau}_{1}} \psi\left(\tau_{1}\right) \int_{0}^{K_{T}-y_{1}} \chi\left(h_{1}\right) \Gamma\left(y_{1}+h_{1}\right) d h_{1} d \tau_{1}
\end{aligned}
$$

When $\bar{\tau}_{1}=\infty$, that is when $y_{0} \leqslant K_{a}, \bar{T}_{1}(t)=0, \forall t$, so that

$$
\hat{p}_{1}\left(s, y_{0}\right)=\int_{0}^{\infty} \psi\left(\tau_{1}\right) \int_{K_{T}-y_{1}}^{\infty} \chi\left(h_{1}\right) e^{-s \tau_{1}} d h_{1} d \tau_{1} .
$$

Using the same token as before, we obtain:

$$
\begin{aligned}
\Gamma\left(y_{0}\right) & =\int_{0}^{\infty} \tau_{1} \psi\left(\tau_{1}\right) d \tau_{1} \\
& +\int_{0}^{\infty} \psi\left(\tau_{1}\right) \int_{0}^{K_{T}-y_{1}} \chi\left(h_{1}\right) \Gamma\left(y_{1}+h_{1}\right) d h_{1} d \tau_{1} .
\end{aligned}
$$

Both (B.17) and (B.19) can be summarized into (B.1).

Considering that catastrophes may occur during the introduction process leads to the following integral equation of the MFPT:

$$
\begin{aligned}
& \Gamma\left(y_{0}\right)=e^{-\lambda_{c} \theta\left(y_{0}\right)}\left(\theta\left(y_{0}\right)+\int_{0}^{\bar{\tau}_{1}} \psi\left(\tau_{1}\right) \int_{0}^{K_{T}-y\left(\theta\left(y_{0}\right)\right)} \chi\left(h_{1}\right) \Gamma\left(y\left(\theta\left(y_{0}\right)\right)+h_{1}\right) d h_{1} d \tau_{1}\right) \\
& +\left(1-e^{-\lambda_{c} \theta\left(y_{0}\right)}\right)\left(\int_{0}^{\infty} \tau_{1} \psi\left(\tau_{1}\right) d \tau_{1}+\int_{0}^{\bar{\tau}_{1}} \psi\left(\tau_{1}\right) \int_{0}^{K_{T}} \chi\left(h_{1}\right) \Gamma\left(h_{1}\right) d h_{1} d \tau_{1}\right) .
\end{aligned}
$$

Differences compared with Equation (9) appear in the double integrals that describe the random timing of introduction events. Further, the mean time to wait for the next event after a catastrophe is now the mean time before an introduction event occurs. 


\section{Appendix C. Successive approximations}

In this part, we prove that the method of successive approximations converges to the integral equation of the MFPT defined by (B.1). Consider that introductions are randomly made and that catastrophes occur, then algorithm equation 10 is written as follows:

$$
\begin{aligned}
& \Gamma_{0}\left(y_{0}\right)=\theta\left(y_{0}\right) \\
& \Gamma_{i+1}\left(y_{0}\right)=e^{-\lambda_{c} \theta\left(y_{0}\right)}\left(\theta\left(y_{0}\right)+\int_{0}^{\bar{\tau}_{1}} \psi\left(\tau_{1}\right) \int_{0}^{K_{T}-y\left(\theta\left(y_{0}\right)\right)} \chi\left(h_{1}\right) \Gamma_{i}\left(y\left(\theta\left(y_{0}\right)\right)+h_{1}\right) d h_{1} d \tau_{1}\right) \\
& +\left(1-e^{-\lambda_{c} \theta\left(y_{0}\right)}\right)\left(\int_{0}^{\infty} \tau_{1} \psi\left(\tau_{1}\right) d \tau_{1}+\int_{0}^{\bar{\tau}_{1}} \psi\left(\tau_{1}\right) \int_{0}^{K_{T}} \chi\left(h_{1}\right) \Gamma_{i}\left(h_{1}\right) d h_{1} d \tau_{1}\right) .
\end{aligned}
$$

Furthermore, we assume that the introduction sizes are defined on $\mathbb{R}_{+}$such that:

$$
\sup \{h \text { such that } \chi(h)>0\}>K_{T} .
$$

This hypothesis supposes that one introduction event may bring the population above the target size $K_{T}$, for all initial density $0 \leqslant y_{0} \leqslant K_{T}$. This hypothesis is satisfied using the log-normal distribution.

Theorem 2. Under Hypothesis (C.2), the integral equation (9) has a unique solution $\Gamma($.$) on A=\mathcal{C}^{0}\left(\left[0, K_{T}\right], \mathbb{R}^{*}\right)$ and successive approximations defined by (C.1) converge to $\Gamma$ (.) for any initialization on $A$.

Proof 2. Using $\|f\|_{\infty}$ as sup $f(x)$, the space $\left(A,\|.\|_{\infty}\right)$ is a Banach space.

Hence, if $\eta$ is a contraction mapping from $A$ to $A$, using Banach's fixed point theorem, the sequence

$$
\Gamma_{i+1}=\eta\left(\Gamma_{i}\right),
$$

with $i \in \mathbb{N}$ and $\eta$ the iteration defined by (C.1), converges to a unique fixed point $E^{*}$ such that $E^{*}=\eta\left(E^{*}\right)$. This fixed point would then be the unique solution of (C.1), which can easily be seen to be the solution of (9).

To prove that (C.3) is a contraction mapping, we compute two sequences, solutions of (C.1) initialized differently. We denote these sequences $\left(\Gamma_{i}\right)_{i \in \mathbb{N}}$ and $\left(\Upsilon_{i}\right)_{i \in \mathbb{N}}$. We will then follow the evolution of

$$
\left\|\eta\left(\Gamma_{i}\right)-\eta\left(\Upsilon_{i}\right)\right\|_{\infty}=\left\|\Gamma_{i+1}-\Upsilon_{i+1}\right\|_{\infty}
$$


We compute this difference between $\Gamma_{i+1}\left(y_{0}\right)$ and $\Upsilon_{i+1}\left(y_{0}\right)$ based on (C.1):

$$
\begin{aligned}
& \Gamma_{i+1}\left(y_{0}\right)-\Upsilon_{i+1}\left(y_{0}\right)= \\
& e^{-\lambda_{c} \theta\left(y_{0}\right)} \int_{0}^{\bar{\tau}_{1}} \psi\left(\tau_{1}\right) \int_{0}^{K_{T}-y\left(\theta\left(y_{0}\right)\right)} \chi\left(h_{1}\right)\left[\Gamma_{i}\left(y\left(\theta\left(y_{0}\right)\right)+h_{1}\right)-\Upsilon_{i}\left(y\left(\theta\left(y_{0}\right)\right)+h_{1}\right)\right] d h_{1} d \tau_{1} \\
& +\left(1-e^{-\lambda_{c} \theta\left(y_{0}\right)}\right) \int_{0}^{\bar{\tau}_{1}} \psi\left(\tau_{1}\right) \int_{0}^{K_{T}} \chi\left(h_{1}\right)\left[\Gamma_{i}\left(h_{1}\right)-\Upsilon_{i}\left(h_{1}\right)\right] d h_{1} d \tau_{1} .
\end{aligned}
$$

The norm of the latter equation is:

$$
\begin{aligned}
& \left\|\eta\left(\Gamma_{i}\right)-\eta\left(\Upsilon_{i}\right)\right\|_{\infty}= \\
& \| e^{-\lambda_{c} \theta\left(y_{0}\right)} \int_{0}^{\bar{\tau}_{1}} \psi\left(\tau_{1}\right) \int_{0}^{K_{T}-y\left(\theta\left(y_{0}\right)\right)} \chi\left(h_{1}\right)\left[\Gamma_{i}\left(y\left(\theta\left(y_{0}\right)\right)+h_{1}\right)-\Upsilon_{i}\left(y\left(\theta\left(y_{0}\right)\right)+h_{1}\right)\right] d h_{1} d \tau_{1} \\
& +\left(1-e^{-\lambda_{c} \theta\left(y_{0}\right)}\right) \int_{0}^{\bar{\tau}_{1}} \psi\left(\tau_{1}\right) \int_{0}^{K_{T}} \chi\left(h_{1}\right)\left[\Gamma_{i}\left(h_{1}\right)-\Upsilon_{i}\left(h_{1}\right)\right] d h_{1} d \tau_{1} \|_{\infty} .
\end{aligned}
$$

Considering that all terms in the integrals are positive, except for $\Gamma_{i}()-$. $\Upsilon_{i}($.$) , we can upper-bound this norm by its value with \left\|\Gamma_{i}-\Upsilon_{i}\right\|_{\infty}$, so that

$$
\begin{aligned}
\left\|\eta\left(\Gamma_{i}\right)-\eta\left(\Upsilon_{i}\right)\right\|_{\infty} & \leq\left\|e^{-\lambda_{c} \theta\left(y_{0}\right)} \int_{0}^{\bar{\tau}_{1}} \psi\left(\tau_{1}\right) \int_{0}^{K_{T}-y\left(\theta\left(y_{0}\right)\right)} \chi\left(h_{1}\right)\right\| \Gamma_{i}-\Upsilon_{i} \|_{\infty} d h_{1} d \tau_{1} \\
& +\left(1-e^{-\lambda_{c} \theta\left(y_{0}\right)}\right) \int_{0}^{\bar{\tau}_{1}} \psi\left(\tau_{1}\right) \int_{0}^{K_{T}} \chi\left(h_{1}\right)\left\|\Gamma_{i}-\Upsilon_{i}\right\|_{\infty} d h_{1} d \tau_{1} \|_{\infty}
\end{aligned}
$$

Then, the terms $\left\|\Gamma_{i}-\Upsilon_{i}\right\|_{\infty}$ can be taken out of the integral.

$$
\begin{aligned}
\left\|\eta\left(\Gamma_{i}\right)-\eta\left(\Upsilon_{i}\right)\right\|_{\infty} & \leq\left\|\Gamma_{i}-\Upsilon_{i}\right\|_{\infty} \cdot \| e^{-\lambda_{c} \theta\left(y_{0}\right)} \int_{0}^{\bar{\tau}_{1}} \psi\left(\tau_{1}\right) \int_{0}^{K_{T}-y\left(\theta\left(y_{0}\right)\right)} \chi\left(h_{1}\right) d h_{1} d \tau_{1} \\
& +\left(1-e^{-\lambda_{c} \theta\left(y_{0}\right)}\right) \int_{0}^{\bar{\tau}_{1}} \psi\left(\tau_{1}\right) \int_{0}^{K_{T}} \chi\left(h_{1}\right) d h_{1} d \tau_{1} \|_{\infty}
\end{aligned}
$$

It is clear that,

$$
\int_{0}^{K_{T}-y\left(\theta\left(y_{0}\right)\right)} \chi\left(h_{1}\right) d h_{1} \leq \int_{0}^{K_{T}} \chi\left(h_{1}\right) d h_{1}
$$


it follows that a simple upper-bound is obtained:

$$
\left\|\eta\left(\Gamma_{i}\right)-\eta\left(\Upsilon_{i}\right)\right\|_{\infty} \leq\left\|\Gamma_{i}-\Upsilon_{i}\right\|_{\infty} \cdot\left\|\int_{0}^{\bar{\tau}_{1}} \psi\left(\tau_{1}\right) \int_{0}^{K_{T}} \chi\left(h_{1}\right) d h_{1} d \tau_{1}\right\|_{\infty} .
$$

By hypothesis C.2 , $\int_{0}^{K_{T}} \chi\left(h_{1}\right) d h_{1}<1$. Further, since $\int_{0}^{\infty} \psi\left(\tau_{1}\right) d \tau_{1}=1$, it follows that the second factor in the right-hand side is strictly smaller than one. Thus, $\eta$ is a contraction mapping. The proof is concluded by using Banach's fixed point theorem. 


\section{Appendix D. Deterministic simulations}
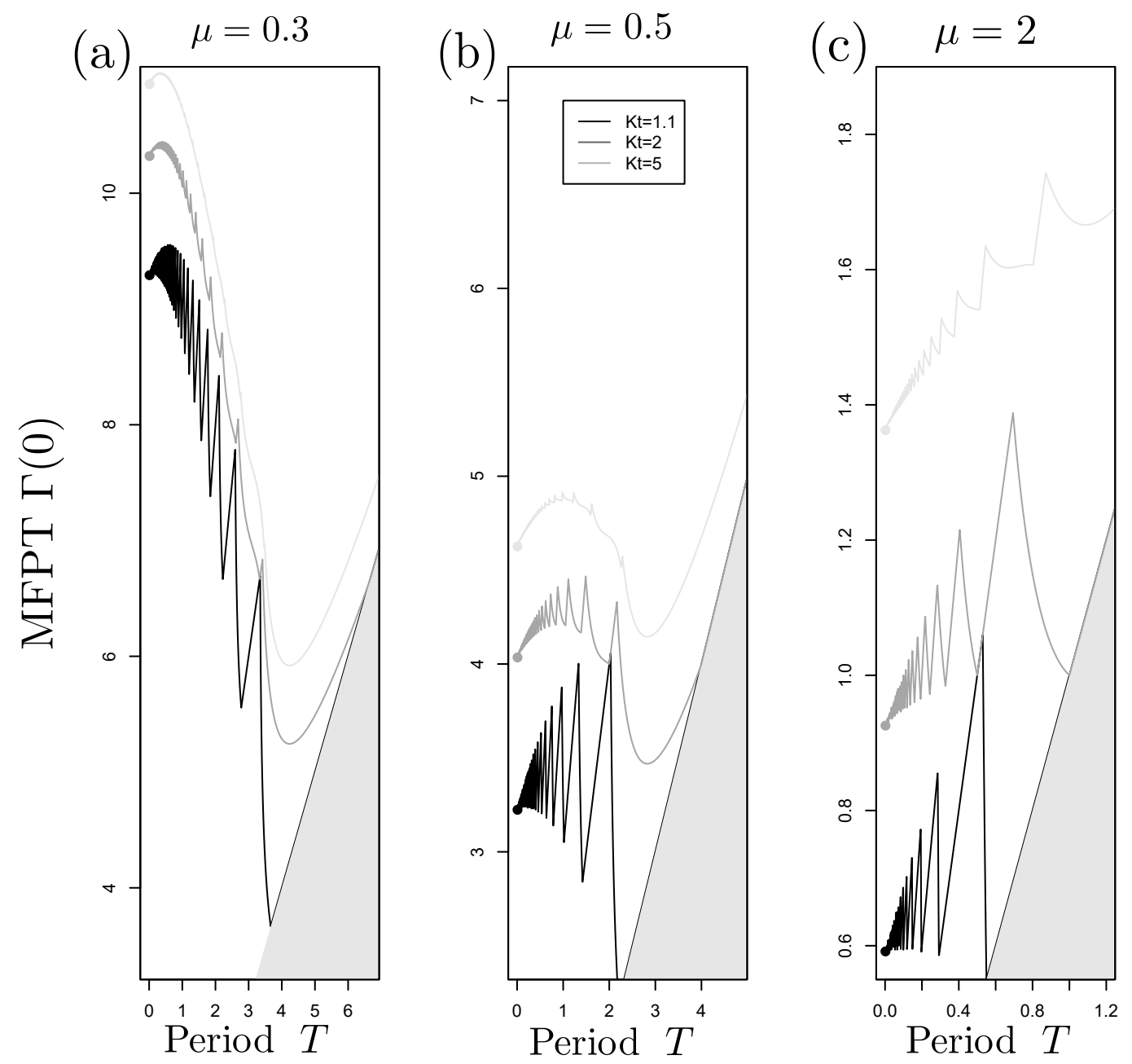

Figure D.7: Influence of $\mu$ and $K_{T}$ : Mean first passage time (MFPT) for population size to cross $K_{T}$ from $y_{0}=0$ as a function of introduction periods. (a) Describes the MFPT for $\mu=0.3$, (b) is obtained using $\mu=0.5$, and (c) for $\mu=2$. Three values of $K_{T}$ are shown: $K_{T}=1.1$ (solid black), $K_{T}=2$ (dark gray) and $K_{T}=5$ (light gray). Points on the left of all sub-figures denote the limit case with the continuous introduction (Model (11)). There is no variability in the introduction sizes, i.e. $c_{p}=0$. Period $T$ varies from 0 to 7 in (a), to 5 in (b), and to 1.2 in (c). MFPT is not reachable in the shaded areas (mean time faster than MFPT in one introduction). 


\section{Appendix E. Monte Carlo simulations}
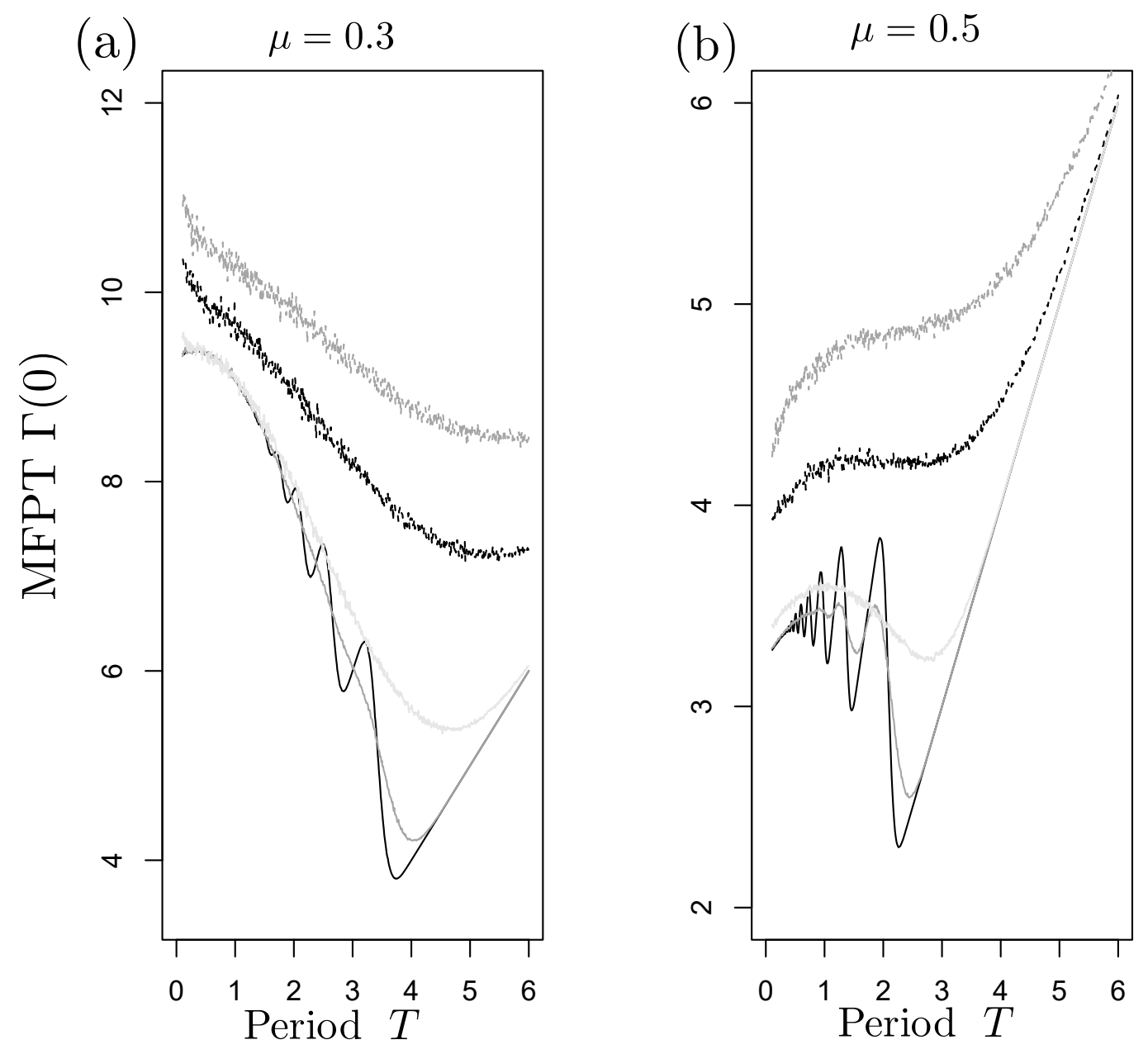

Figure E.8: Influence of $\mu$ and $c_{p}$ : Mean first passage time (MFPT) using the Monte Carlo method for different values of the coefficient of proportionality between the variance and the mean introduction size. (a) The MFPT for an introduction rate $\mu=0.3$, (b) is for $\mu=0.5$. The MFPT is given with respect to the period of introduction for a fixed value of $y_{0}=0$. The solid and dashed curves describe the MFPT for different variability in introduction sizes for all sub-figures. Solid black, dark, and light gray show the MFPT for small variances with $c_{p}=0.001, c_{p}=0.01$ and $c_{p}=0.1$, respectively. Dashed black and dark gray show the MFPT for larger variances using $c_{p}=0.5, c_{p}=1$, respectively.

Figure E.8 represents the MFPT curves with the same parameters used 
in Figure 4, but using Monte Carlo simulations: we computed 10000 simulations with an initial population at $y_{0}=0$ and computed the mean time to cross the target size. This figure shows that all patterns observed in Figure 4 are the same compared to this alternative approach to computing the MFPT, which takes much longer time to obtain (roughly from 5 to 100 times longer). Further, with Monte Carlo simulations, only one MFPT is computed from $y_{0}=0$, while it was computed from $y_{0}=0$ to $y_{0}=K_{T}$ using the successive approximation method.

\section{References}

Amarasekare P. Allee effects in metapopulation dynamics. The American Naturalist 1998;152:298-302.

Armstrong DP, Seddon PJ. Directions in reintroduction biology. Trends in Ecology \& Evolution 2008;23(1):20-5.

Bajeux N, Grognard F, Mailleret L. Introduction strategies for biological control agents subject to allee effects. In: 21st International Symposium on Mathematical Theory of Networks and Systems. 2014. .

Bajeux N, Grognard F, Mailleret L. Augmentative biocontrol when natural enemies are subject to allee effects. Journal of Mathematical Biology 2017;74(7):1561-87.

Bascompte J. Extinction thresholds: insights from simple models. In: Annales Zoologici Fennici. JSTOR; 2003. p. 99-114.

Blackburn TM, Duncan RP. Establishment patterns of exotic birds are constrained by non-random patterns in introduction. Journal of Biogeography 2001;28(7):927-39.

Blackburn TM, Pyšek P, Bacher S, Carlton JT, Duncan RP, Jarošík V, Wilson JR, Richardson DM. A proposed unified framework for biological invasions. Trends in ecology \& evolution 2011;26(7):333-9.

Boukal DS, Berec L. Single-species models of the allee effect: extinction boundaries, sex ratios and mate encounters. Journal of Theoretical Biology $2002 ; 218(3): 375-94$. 
Brassil CE. Mean time to extinction of a metapopulation with an allee effect. Ecological Modelling 2001;143(1):9-16.

Cassey P, Prowse TA, Blackburn TM. A population model for predicting the successful establishment of introduced bird species. Oecologia 2014;175(1):417-28.

Courchamp F, Clutton-Brock T, Grenfell B. Inverse density dependence and the allee effect. Trends in Ecology and Evolution 1999;14:405-10.

Dennis B. Allee effects in stochastic populations. Oikos 2002;96(3):389-401.

Dennis B, Assas L, Elaydi S, Kwessi E, Livadiotis G. Allee effects and resilience in stochastic populations. Theoretical ecology 2016;9(3):323-35.

Deredec A, Courchamp F. Importance of the allee effect for reintroductions. Ecoscience 2007;14(4):440-51.

Dlugosch KM, Parker IM. Founding events in species invasions: genetic variation, adaptive evolution, and the role of multiple introductions. Molecular Ecology 2008;17(8):431-49.

Drake DAR, Casas-Monroy O, Koops MA, Bailey SA. Propagule pressure in the presence of uncertainty: extending the utility of proxy variables with hierarchical models. Methods in Ecology and Evolution 2015;6(11):136371 .

Drake JM, Baggenstos P, Lodge DM. Propagule pressure and persistence in experimental populations. Biology Letters 2005;1(4):480-3.

Drake JM, Lodge DM. Allee effects, propagule pressure and the probability of establishment: risk analysis for biological invasions. Biological Invasions 2006;8(2):365-75.

Drolet D, Locke A, et al. Relative importance of propagule size and propagule number for establishment of non-indigenous species: a stochastic simulation study. Aquatic Invasions 2016;11(1):101-10.

Drury K, Drake J, Lodge D, Dwyer G. Immigration events dispersed in space and time: Factors affecting invasion success. ecological modelling 2007;206(1):63-78. 
Drury KL. Shot noise perturbations and mean first passage times between stable states. Theoretical population biology 2007;72(1):153-66.

Fauvergue X, Vercken E, Malausa T, Hufbauer RA. The biology of small, introduced populations, with special reference to biological control. Evolutionary applications 2012;5(5):424-43.

Grevstad FS. Factors influencing the chance of population establishment: implications for release strategies in biocontrol. Ecological Applications 1999;9(4):1439-47.

Gruntfest Y, Arditi R, Dombrovsky Y. A fragmented population in a varying environment. Journal of Theoretical Biology 1997;185(4):539-47.

Haccou P, Iwasa Y. Establishment probability in fluctuating environments: a branching process model. Theoretical population biology 1996;50(3):25480 .

Hedge LH, O'Connor WA, Johnston EL. Manipulating the intrinsic parameters of propagule pressure: implications for bio-invasion. Ecosphere $2012 ; 3(6): 1-13$.

Jerde CL, Lewis MA. Waiting for invasions: a framework for the arrival of nonindigenous species. The American Naturalist 2007;170(1):1-9.

Lande R. Risks of population extinction from demographic and environmental stochasticity and random catastrophes. The American Naturalist 1993;142(6):911-27.

Lande R, Engen S, Saether BE. Stochastic population dynamics in ecology and conservation. Oxford University Press on Demand, 2003.

van Lenteren JC. Success in biological control of arthropods by augmentation of natural enemies. In: Biological Control: Measures of Success. Springer; 2000. .

Lewis M, Kareiva P. Allee dynamics and the spread of invading organisms. Theoretical Population Biology 1993;43(2):141-58.

Lewis M, Petrovskii S, Potts J. Stochasticity and invasion dynamics. In: The mathematics behind biological invasions. Springer; volume 44 of Interdisciplinary applied mathematics; 2016. . 
Liebhold A, Bascompte J. The allee effect, stochastic dynamics and the eradication of alien species. Ecology Letters 2003;6(2):133-40.

Lockwood JL, Cassey P, Blackburn T. The role of propagule pressure in explaining species invasions. Trends in Ecology \& Evolution 2005;20(5):2238.

Mailleret L, Grognard F. Optimal release policy for prophylactic biological control. Positive systems 2006;:89-96.

Mailleret L, Grognard F. Global stability and optimisation of a general impulsive biological control model. Mathematical Biosciences 2009;221:91 $-100$.

Mailleret L, Lemesle V. A note on semi-discrete modelling in the life sciences. Philosophical Transactions of the Royal Society A-Mathematical Physical and Engineering Sciences 2009;367:2113-38.

Masoliver J. First-passage times for non-markovian processes: Shot noise. Physical Review A 1987;35(9):3918.

Nundloll S, Mailleret L, Grognard F. Two models of interfering predators in impulsive biological control. Journal of Biological Dynamics 2010;4(1):10214.

Polizzi NF, Therien MJ, Beratan DN. Mean first-passage times in biology. Israel journal of chemistry 2016;56(9-10):816-24.

Polyanin AD, Manzhirov AV. Handbook of integral equations. CRC press, 2008.

Potapov A, Rajakaruna H. Allee threshold and stochasticity in biological invasions: Colonization time at low propagule pressure. Journal of theoretical biology 2013;337:1-14.

Rajakaruna H, Potapov A, Lewis M. Impact of stochasticity in immigration and reintroduction on colonizing and extirpating populations. Theoretical population biology 2013;85:38-48.

Shaffer M. Minimum viable populations: coping with uncertainty. Viable populations for conservation 1987;69:86. 
Shestopalov YV, Smirnov YG. Integral equations. Karlstadt UniversityDivision for engineer sciences, physics and mathematics, Karlstadt 2002;

Simberloff D. The role of propagule pressure in biological invasions. Annual Review of Ecology, Evolution, and Systematics 2009;40:81-102.

Sinclair JS, Arnott SE. Strength in size not numbers: propagule size more important than number in sexually reproducing populations. Biological Invasions 2016;:1-9.

Taylor CM, Hastings A. Allee effects in biological invasions. Ecology Letters 2005;8(8):895-908.

Tobin PC, Berec L, Liebhold AM. Exploiting allee effects for managing biological invasions. Ecology letters 2011;14(6):615-24.

Van Kampen NG. Stochastic processes in physics and chemistry. volume 1. Elsevier, 1992.

Wang MH, Kot M. Speeds of invasion in a model with strong or weak allee effects. Mathematical biosciences 2001;171(1):83-97.

Wittmann MJ, Metzler D, Gabriel W, Jeschke JM. Decomposing propagule pressure: the effects of propagule size and propagule frequency on invasion success. Oikos 2014;123(4):441-50. 